TRANSACTIONS OF THE

AMERICAN MATHEMATICAL SOCIETY

Volume 354, Number 10, Pages 4201-4227

S 0002-9947(02)03033-7

Article electronically published on May 15, 2002

\title{
SCHRÖDINGER OPERATORS WITH NON-DEGENERATELY VANISHING MAGNETIC FIELDS IN BOUNDED DOMAINS
}

\author{
XING-BIN PAN AND KENG-HUAT KWEK
}

\begin{abstract}
We establish an asymptotic estimate of the lowest eigenvalue $\mu(b \mathbf{F})$ of the Schrödinger operator $-\nabla_{b \mathbf{F}}^{2}$ with a magnetic field in a bounded 2dimensional domain, where curl $\mathbf{F}$ vanishes non-degenerately, and $b$ is a large parameter. Our study is based on an analysis on an eigenvalue variation problem for the Sturm-Liouville problem. Using the estimate, we determine the value of the upper critical field for superconductors subject to non-homogeneous applied magnetic fields, and localize the nucleation of superconductivity.
\end{abstract}

\section{$\S 1$. INTRODUCTION}

In this paper we study the lowest eigenvalue $\mu(b \mathbf{F})$ for the Schrödinger operator $-\nabla_{b \mathbf{F}}^{2}$ with a magnetic field $\mathbf{F}$, where curl $\mathbf{F}$ vanishes non-degenerately in a bounded 2-dimensional domain, and derive asymptotic estimates for the large parameter $b$. This problem arises in the mathematical theory of describing the nucleation phenomenon for superconductors subject to non-homogeneous applied magnetic fields. Using the estimates of $\mu(b \mathbf{F})$, we determine the value of the upper critical field and the location of nucleation of superconductivity. This problem is interesting to us also because of its connection to the problem of estimating the lowest eigenvalue and describing the (bounded) eigenfunctions ${ }^{1}$ of the Schrödinger operator with a non-degenerately vanishing magnetic field in the entire plane $\mathbb{R}^{2}$ and in the half-plane $\mathbb{R}_{+}^{2}$.

A type 2 superconductor subject to an applied magnetic field will exhibit many interesting phenomena. It is well-known that, if the applied magnetic field is homogeneous and decreases from the upper critical value $H_{C_{3}}$, superconductivity nucleates at the surface of the sample. The estimate of the upper critical field and the localization of the nucleation of superconductivity have been studied by many physicists; see Saint-James and De Gennes [SdG], Saint-James and Sarma [SST], and Tinkham T]. More recently, a mathematical analysis of these problems has been carried out by many mathematicians. Among them we would like to mention the work of Chapman [C] and Bernoff-Sternberg [BS] based on the formal

Received by the editors July 17, 2000 and, in revised form, March 13, 2001.

2000 Mathematics Subject Classification. Primary 35Q55, 81Q10, 82D55.

Key words and phrases. Schrödinger operator with a magnetic field, eigenvalue, GinzburgLandau system, superconductivity, nucleation, upper critical field, Sturm-Liouville operator, Riccati type equation.

${ }^{1}$ In this paper, for convenience, when considering eigenvalue problems in the entire plane or in the half-plane, we call a non-trivial bounded solution an eigenfunction. Therefore, the eigenfunctions associated with the lowest eigenvalue need not lie in $L^{2}$. 
analysis, Bauman-Phillips-Tang [BPT] for the rigorous analysis on disks, GiorgiPhillips [GP, Lu-Pan LP1], LP2, [LP3, [LP4, LP5], LP6], del Pino-FelmerSternberg [DFS], Pan [P], Helffer-Morame [HMor] and Helffer-Pan [HP] for rigorous analysis on general domains.

To study the nucleation of superconductivity for a sample with large value of the Ginzburg-Landau parameter $\kappa$, and subject to a strong magnetic field $\mathcal{H}_{\text {appl }} \equiv$ $\mathcal{H}=\sigma \mathbf{H}_{0}$ with large $\sigma$, Lu and Pan [LP4], [LP5] introduced a number $\sigma^{*}(\kappa)$, which depends on $\mathbf{H}_{0}$, such that the sample is in the normal state if $\sigma>\sigma^{*}(\kappa)$ and is in the superconducting state if $0 \leq \sigma<\sigma^{*}(\kappa)$. In the special case when the applied field is homogeneous, $\sigma^{*}(\kappa)$ is equal to the upper-critical field $H_{C_{3}}$. It was shown that, for a bounded sample with smooth surface, we have

$$
\lim _{\kappa \rightarrow+\infty} \frac{\sigma^{*}(\kappa)}{\kappa}=\frac{1}{\alpha_{0}\left(\mathbf{H}_{0}\right)}
$$

where $\alpha_{0}\left(\mathbf{H}_{0}\right)$ is a number determined by $\Omega$ and $\mathbf{H}_{0}$. In the special case where the applied field is homogeneous, the above equality yields an estimate for $H_{C_{3}}$.

Although in [LP1], [LP2], [LP3], [LP4], [LP5] the authors mainly discussed the case where the applied field does not vanish, it was observed in LP3] (Proposition 6.3) that the value of $\sigma^{*}(\kappa)$ is greatly raised if $\mathbf{H}_{0}$ has zeros: $\sigma^{*}(\kappa) \geq C \kappa^{2}$ for all large $\kappa$. In this paper we shall study this phenomenon more precisely. Let us consider a cylindrical superconductor with simply-connected cross section $\Omega$, and placed in an applied magnetic field which is parallel to the lateral surface. Choose the axis of the cylinder as the $x_{3}$-axis. Then $\Omega$ is a bounded domain in $\mathbb{R}^{2}$. In this paper we always assume that

$$
\mathcal{H}=H \mathbf{e}_{3}, \quad H=\sigma H_{0},
$$

where $H_{0}$ is a smooth function of $x=\left(x_{1}, x_{2}\right)$, and $\mathbf{e}_{3}$ is the unit vector along the positive $x_{3}$-axis. Then, superconductivity can be described by a complex-valued order parameter $\psi$ and a magnetic potential $\mathcal{A}$, and $(\psi, \mathcal{A})$ is a minimizer of the Ginzburg-Landau functional. The Euler equation is the 2-dimensional GinzburgLandau system (see [GL, dG], SST, CHO], [DGP]):

$$
\begin{cases}-(\nabla-i \kappa \mathcal{A})^{2} \psi=\kappa^{2}\left(1-|\psi|^{2}\right) \psi & \\ \operatorname{curl}^{2} \mathcal{A}=-\frac{i}{2 \kappa}(\bar{\psi} \nabla \psi-\psi \nabla \bar{\psi})-|\psi|^{2} \mathcal{A}+\operatorname{curl} H, & \text { in } \Omega \\ \frac{\partial \psi}{\partial \nu}-i \kappa \mathcal{A} \psi \cdot \nu+\gamma \psi=0, & \text { on } \partial \Omega \\ \operatorname{curl} \mathcal{A}-H=0, & \text { on }\end{cases}
$$

Here $i=\sqrt{-1}, \kappa$ is the Ginzburg-Landau parameter and $\gamma \geq 0$. $\gamma$ is very small for an insulator, very large for magnetic material, and in between for non-magnetic material.

As we consider strong fields $H=\sigma H_{0}$ with large $\sigma$, in the following, we set

$$
\mathcal{A}=\sigma \mathbf{A} \text {. }
$$

We rewrite the Ginzburg-Landau functional as follows:

$$
\mathcal{E}(\psi, \mathbf{A})=\int_{\Omega}\left\{\left|\nabla_{\sigma \kappa \mathbf{A}} \psi\right|^{2}+(\sigma \kappa)^{2}\left|\operatorname{curl} \mathbf{A}-H_{0}\right|^{2}+\frac{\kappa^{2}}{2}\left(|\psi|^{2}-1\right)^{2}\right\} d x+\int_{\partial \Omega} \gamma|\psi|^{2} d s
$$


In the 2-dimensional case, $\mathbf{A}=\left(A_{1}, A_{2}\right)$, and

$$
\begin{gathered}
\nabla_{\mathbf{A}} \psi=\nabla \psi-i \mathbf{A} \psi, \quad \operatorname{curl} \mathbf{A}=\partial_{1} A_{2}-\partial_{2} A_{1}, \quad \operatorname{curl}^{2} \mathbf{A}=\left(\partial_{2} \operatorname{curl} \mathbf{A},-\partial_{1} \operatorname{curl} \mathbf{A}\right), \\
\nabla_{\mathbf{A}}^{2} \psi=(\nabla-i \mathbf{A})^{2} \psi=\Delta \psi-i[2 \mathbf{A} \cdot \nabla \psi+\psi \operatorname{div} \mathbf{A}]-|\mathbf{A}|^{2} \psi .
\end{gathered}
$$

To state our main results, we need a few definitions and notations. Given $H_{0}$, let

$$
\mathcal{Z}\left(H_{0}\right)=\left\{x \in \bar{\Omega}: H_{0}(x)=0\right\} .
$$

We say that $H_{0}$ vanishes non-degenerately if the zero set $\mathcal{Z}\left(H_{0}\right)$ satisfies the following condition:

$\mathcal{Z}\left(H_{0}\right)$ is the union of a finite number of smooth curves and $\nabla H_{0} \neq 0$ on $\mathcal{Z}\left(H_{0}\right)$.

For the given function $H_{0}$, there exists a unique smooth vector field $\mathbf{F}$ on $\bar{\Omega}$ such that

$$
\operatorname{curl} \mathbf{F}=H_{0} \quad \text { and } \quad \operatorname{div} \mathbf{F}=0 \quad \text { in } \Omega, \quad \mathbf{F} \cdot \nu=0 \quad \text { on } \partial \Omega .
$$

Note that $(0, \mathbf{F})$ is a trivial critical point of $\mathcal{E}$ for any $\sigma$. Let us recall the definition of $\sigma^{*}\left(\kappa, H_{0}\right)$ given in [LP4] (Section 1):

$$
\sigma^{*}\left(\kappa, H_{0}\right)=\inf \{\sigma>0:(0, \mathbf{F}) \text { is the only minimizer of } \mathcal{E}\} .
$$

We shall estimate $\sigma^{*}\left(\kappa, H_{0}\right)$ for non-degenerately vanishing $H_{0}$.

Throughout this paper we assume that $\Omega$ is a bounded smooth $\left(C^{3}\right)$ and simplyconnected domain in $\mathbb{R}^{2}$. We denote the unit outward normal of $\partial \Omega$ by $\nu=\left(\nu_{1}, \nu_{2}\right)$ and the unit tangential vector by $\tau=\left(\tau_{1}, \tau_{2}\right)=\left(-\nu_{2}, \nu_{1}\right)$. We choose the orientation of $\partial \Omega$ so that the orientation of the $\{\mu, \tau\}$ is the same as that of the $x_{1} x_{2}$-coordinate system. For $x \in \partial \Omega$, denote by $\vartheta(x)$ the angle between the vector

$\operatorname{curl}^{2} \mathbf{F}(x)$ and $\tau$. Note that $\vartheta(x)$ is equal to the angle between $\nabla H_{0}(x)$ and the inward normal vector $-\nu(x)$. Define

$$
\mathcal{Z}\left(H_{0}, \Omega\right)=\mathcal{Z}\left(H_{0}\right) \cap \Omega, \quad \mathcal{Z}\left(H_{0}, \partial \Omega\right)=\mathcal{Z}\left(H_{0}\right) \cap \partial \Omega .
$$

Let $\lambda_{0}$ be the number given in $(1.10)$ below, and let $\lambda\left(\mathbb{R}^{2}, \vartheta\right)$ be the lowest eigenvalue of the eigenvalue problem (1.11). Define

$$
\alpha_{1}\left(H_{0}\right)=\min \left\{\lambda_{0}^{3 / 2} \min _{x \in \mathcal{Z}\left(H_{0}, \Omega\right)}\left|\nabla H_{0}(x)\right|, \min _{x \in \mathcal{Z}\left(H_{0}, \partial \Omega\right)} \lambda\left(\mathbb{R}_{+}^{2}, \vartheta(x)\right)^{3 / 2}\left|\nabla H_{0}(x)\right|\right\} .
$$

We also define

$$
\begin{aligned}
\tilde{\mathcal{Z}}\left(H_{0}\right)= & \left\{x \in \Omega: \lambda_{0}^{3 / 2}\left|\nabla H_{0}(x)\right|=\alpha_{1}\left(H_{0}\right)\right\} \\
& \cup\left\{x \in \partial \Omega: \lambda\left(\mathbb{R}_{+}^{2}, \vartheta(x)\right)^{3 / 2}\left|\nabla H_{0}(x)\right|=\alpha_{1}\left(H_{0}\right)\right\} .
\end{aligned}
$$

Note that if all the zero points of $H_{0}$ lie inside $\Omega$, then

$$
\tilde{\mathcal{Z}}\left(H_{0}\right)=\left\{x \in \Omega: H_{0}(x)=0, \quad\left|\nabla H_{0}(x)\right|=\min _{y \in \mathcal{Z}(\Omega)}\left|\nabla H_{0}(y)\right|\right\} .
$$

Theorem 1. Assume that condition (1.1) is satisfied.

(i) Asymptotic estimate for $\sigma^{*}\left(\kappa, H_{0}\right)$ :

$$
\lim _{\kappa \rightarrow+\infty} \frac{\sigma^{*}\left(\kappa, H_{0}\right)}{\kappa^{2}}=\frac{1}{\alpha_{1}\left(H_{0}\right)} .
$$


(ii) Concentration of order parameters: Let $\left\{k_{n}\right\}$ and $\left\{\sigma_{n}\right\}$ be such that

$$
\kappa_{n} \rightarrow+\infty, \quad \sigma_{n}<\sigma^{*}\left(\kappa_{n}, H_{0}\right), \quad \frac{\sigma_{n}}{\kappa_{n}^{2}} \rightarrow \frac{1}{\alpha_{1}\left(H_{0}\right)} \quad \text { as } n \rightarrow \infty .
$$

Let $\left(\psi_{n}, \mathbf{A}_{n}\right)$ be a non-trivial minimizer of the Ginzburg-Landau functional $\mathcal{E}$ with $\kappa=\kappa_{n}$ and $\sigma=\sigma_{n}$. Then, as $n \rightarrow \infty$, we have

$$
\begin{aligned}
& \left\|\psi_{n}\right\|_{L^{\infty}(\Omega)} \rightarrow 0 \quad \text { and } \quad \operatorname{curl} \mathbf{A}_{n} \rightarrow H_{0} \quad \text { in } C^{\alpha}(\Omega), \\
& \frac{\psi_{n}(x)}{\left\|\psi_{n}\right\|_{L^{\infty}(\Omega)}} \rightarrow 0 \quad \text { on } \bar{\Omega} \backslash \tilde{\mathcal{Z}}\left(H_{0}\right) .
\end{aligned}
$$

Remark 1.1. (1) Under the conditions of Theorem 1, we can use the arguments in LP4 (proof of Theorem 1.3) to show that, after rescaling and gauge transformations, a subsequence of $\left\{\psi_{n} /\left\|\psi_{n}\right\|_{L^{\infty}}\right\}$ converges to an eigenfunction of either (1.6) or (1.7) given below.

(2) From Theorem 1 we see that superconductivity persists in a non-degenerately vanishing applied magnetic field up to the level $O\left(\kappa^{2}\right)$. In a decreasing field $H=\sigma H_{0}$ with non-degenerate zeros, superconductivity nucleates at some zero points of $H_{0}$, namely, the set $\tilde{\mathcal{Z}}\left(H_{0}\right)$. If all the zero points of $H_{0}$ lie inside $\Omega$, then nucleation occurs first at the zero points of $H_{0}$ where the magnitude of the gradient $\nabla H_{0}$ is the least.

(3) The geometry of the zero set $\mathcal{Z}\left(H_{0}\right)$ of the magnetic field $H_{0}$ has an effect on the value of $\sigma^{*}\left(\kappa, H_{0}\right)$ and on the localization of the order parameters. We believe that, if the gap $\sigma^{*}\left(\kappa, H_{0}\right)-\sigma$ is sufficiently small, then the order parameters will concentrate at a subset $\Gamma$ of $\tilde{\mathcal{Z}}\left(H_{0}\right)$; and if $\sigma^{*}\left(\kappa, H_{0}\right)-\sigma$ is not very small, then the order parameters will localize uniformly on $\tilde{\mathcal{Z}}\left(H_{0}\right)$. It is interesting to find the geometric characterization of $\Gamma$. For this purpose, one has to estimate the difference

$$
\sigma^{*}\left(\kappa, H_{0}\right)-\frac{\kappa^{2}}{\alpha_{1}\left(H_{0}\right)}
$$

for large $\kappa$. To obtain an upper bound for $\sigma^{*}\left(\kappa, H_{0}\right)-\frac{\kappa^{2}}{\alpha_{1}\left(H_{0}\right)}$, one may follow the method in [LP4] (Appendix). The description of eigenfunctions for (1.6) and (1.11) given in Theorems 3 and 4 will be useful. One may also use the method in $[\mathrm{HP}]$ to derive a lower bound of $\sigma^{*}\left(\kappa, H_{0}\right)-\frac{\kappa^{2}}{\alpha_{1}\left(H_{0}\right)}$, and to discuss the localization of order parameters.

(4) It could be interesting to study similar problems in dimension 3, and study applied fields with higher-order zeros. We would like to mention that the semiclassical analysis for the bottom of the spectrum of the Schrödinger operator with a magnetic field vanishing up to higher order in the entire space $\mathbb{R}^{n}$ has been carried out by Montgomery [M] and Helffer and Mohamed HMoh (see H2 for a review).

As was observed in [LP4] and [LP5], the estimate of $\sigma^{*}\left(\kappa, H_{0}\right)$ and the localization of the nucleation phenomena rely on the estimate of the lowest eigenvalue $\mu(b \mathbf{F})$ of the Schrödinger operator $-\nabla_{b \mathbf{F}}^{2}$ on $\Omega$ for a large parameter $b$ :

$$
-\nabla_{b \mathbf{F}}^{2} \phi=\mu \phi \text { in } \Omega, \quad\left(\nabla_{b \mathbf{F}} \phi\right) \cdot \nu+\gamma \phi=0 \text { on } \partial \Omega .
$$

In fact, for a magnetic field with non-degenerate zeros, we can show that

$$
\lim _{\kappa \rightarrow+\infty} \frac{\sigma^{*}\left(\kappa, H_{0}\right)}{\kappa^{2}}=\lim _{\kappa \rightarrow+\infty} \frac{\sigma_{*}\left(\kappa, H_{0}\right)}{\kappa^{2}},
$$


where

$$
\sigma_{*}\left(\kappa, H_{0}\right)=\min \left\{\sigma>0: \mu(\sigma \kappa \mathbf{F})=\kappa^{2}\right\} .
$$

Therefore, the following theorem is essential to establish Theorem 1.

Theorem 2. Let $H_{0}$ satisfy condition (1.1) and let the vector field $\mathbf{F}$ be determined by (1.2). Then

$$
\lim _{b \rightarrow \infty} \frac{\mu(b \mathbf{F})}{|b|^{2 / 3}}=\alpha_{1}\left(H_{0}\right)^{2 / 3},
$$

where $\alpha_{1}\left(H_{0}\right)$ was given in (1.3). Let $\psi_{b}$ be the eigenfunction of (1.5) associated with the lowest eigenvalue $\mu(b \mathbf{F})$. Then

$$
\lim _{b \rightarrow \infty} \frac{\psi_{b}(x)}{\left\|\psi_{b}\right\|_{L^{\infty}(\bar{\Omega})}}=0 \quad \text { for all } x \in \bar{\Omega} \backslash \tilde{\mathcal{Z}}\left(H_{0}\right) .
$$

Remark 1.2. (1) Theorem 2 says that

$$
\mu(b \mathbf{F})=\left[\alpha_{1}\left(H_{0}\right)^{2 / 3}+o(1)\right]|b|^{2 / 3} \quad \text { as } b \rightarrow \infty .
$$

Using the Helffer-Morame method [HMor], one may obtain a better estimate of the remainder.

(2) It is interesting to find the localization of the eigenfunctions of (1.5) for large $b$, and explore the effect of the geometry of the zero set of $H_{0}$.2 Using the Agmon estimates [A] and the Helffer-Morame method [HMor], one can show that, in the $L^{2}$ sense, the eigenfunctions concentrate at $\tilde{\mathcal{Z}}\left(H_{0}\right)$, and decay exponentially in the normal direction to $\mathcal{Z}\left(H_{0}\right)$.

In the following, we shall describe the main ideas in our approach, and the technical problems we shall solve.

As in [LP1], [LP3, [LP5, the asymptotic estimate for $\mu(b \mathbf{F})$ for large $b$ will be given in terms of the lowest eigenvalues of the Schrödinger operator $-\nabla_{\mathbf{A}}^{2}$ in the plane $\mathbb{R}^{2}$ (when $\mathcal{Z}\left(H_{0}, \Omega\right) \neq \emptyset$ ):

$$
-\nabla_{\mathbf{A}}^{2} \psi=\lambda \psi \quad \text { in } \mathbb{R}^{2}
$$

and on the half plane $\mathbb{R}_{+}^{2}=\left\{\left(x_{1}, x_{2}\right): x_{2}>0\right\}\left(\right.$ when $\left.\mathcal{Z}\left(H_{0}, \partial \Omega\right) \neq \emptyset\right)$ :

$$
-\nabla_{\mathbf{A}}^{2} \varphi=\lambda \varphi \text { in } \mathbb{R}_{+}^{2}, \quad\left(\nabla_{\mathbf{A}} \varphi\right) \cdot \nu=0 \text { on } \partial \mathbb{R}_{+}^{2},
$$

where

$$
\mathbf{A}=-\frac{|x|^{2}}{2} \mathbf{n}, \quad \mathbf{n}=(\cos \vartheta, \sin \vartheta)
$$

Note that curl $\mathbf{A}=x_{2} \cos \vartheta-x_{1} \sin \vartheta$, which vanishes along a line.

In the entire plane case, we can simplify (1.6) by first rotating the coordinate system so that $\mathbf{n}$ is in the positive $y_{1}$ direction, then making a gauge transformation (see Section 4 for the details). In the new coordinate system we may assume $\mathbf{n}=(1,0)$ and $\mathbf{A}=\left(-\frac{1}{2} y_{2}^{2}, 0\right)$. Equation (1.6) is then written as

$$
-\Delta \psi-i y_{2}^{2} \partial_{1} \psi+\frac{1}{4} y_{2}^{4} \psi=\lambda \psi \quad \text { in } \mathbb{R}^{2} .
$$

Eigenvalue problems in the entire plane $\mathbb{R}^{2}$ including (1.6) have been studied by Montgomery [M], Helffer and Mohamed [HMoh], and Helffer [H2]. Among other things, Montgomery showed that, for the magnetic field $\mathbf{A}$ given above, the lowest

\footnotetext{
${ }^{2}$ See Remark 1.1 (3) for a related problem of localization of order parameters.
} 
eigenvalue $\lambda\left(\mathbb{R}^{2}, \mathbf{A}\right)$ of (1.6) is equal to the minimum of $\lambda(\tau)$. Here, for fixed $\tau$, $\lambda(\tau)$ is the lowest eigenvalue of the differential operator $-\frac{d^{2}}{d t^{2}}+\frac{1}{4}\left(t^{2}+2 \tau\right)^{2}$ :

$$
-y^{\prime \prime}+\frac{1}{4}\left(t^{2}+2 \tau\right)^{2} y=\lambda(\tau) y, \quad-\infty<t<\infty .
$$

Namely,

$$
\lambda\left(\mathbb{R}^{2}, \mathbf{A}\right)=\lambda_{0},
$$

where

$$
\lambda_{0}=\inf _{\tau \in \mathbb{R}} \lambda(\tau) .
$$

The numerical computations shows that (see $[\mathrm{M}],[\mathrm{H} 2]) \lambda_{0} \simeq 0.5698$. More general results in higher dimensional spaces were obtained by Helffer and Mohamed [HMoh] and Helffer [H2]. The asymptotic estimates of the lowest eigenvalue and the localization of the eigenfunctions were obtained there.

In this paper we shall classify the eigenfunctions of (1.8) associated with $\lambda\left(\mathbb{R}^{2}, \mathbf{A}\right)$. We shall see that the key point in the classification of eigenfunctions is the uniqueness of the minimum points of $\lambda(\tau)$. So we ask the following two questions:

(Q1) Are the minimum points of the function $\lambda(\tau)$ unique?

(Q2) Are the eigenfunctions of $\lambda\left(\mathbb{R}^{2}, \mathbf{A}\right)$ uniquely determined (up to gauge transformations, rotations and translations) by the eigenfunction $u$ of (1.9) with $\tau=\tau_{0}$ ?

We mention that the answers to these questions are needed to solve the eigenvalue problem (1.7) in the half plane (see Section 5), and will also be needed to describe the behavior of the order parameter and to get higher-order estimates for $\sigma^{*}\left(\kappa, H_{0}\right)$. Note that numerical results suggest that the minimum points of $\lambda(\tau)$ are unique (see [H2]).

Theorem 3. (i) The minimum point $\tau_{0}$ of $\lambda(\tau)$ is unique.

(ii) Let $u$ be the positive eigenfunction of (1.9) with $\tau=\tau_{0}$. The eigenfunctions of (1.8) associated with the lowest eigenvalue $\lambda\left(\mathbb{R}^{2}, \mathbf{A}\right)$ are given by $\psi=c e^{i \tau_{0} y_{1}} u\left(y_{2}\right)$.

Remark 1.3. Note that the zero set of curl $\mathbf{A}$ is unbounded, and the magnitude $|\psi|$ of the eigenfunction $\psi$ is constant along the direction of $\mathbf{n}=(1,0)$. It will be interesting to compare this fact with a result of Helffer and Mohamed ([HMoh], Theorem 2.1), which says that the eigenfunctions of a Schrödinger operator with a magnetic field which is bounded away from zero outside a ball decay exponentially at infinity.

Next we consider (1.7). In contrast to the entire plane case, the lowest eigenvalue of (1.7) depends on the direction of $\mathbf{n}$. After gauge transformation, (1.7) can be written as

$$
\left\{\begin{array}{l}
-\Delta \varphi-i\left(x_{2}^{2} \cos \vartheta \partial_{1} \varphi+x_{1}^{2} \sin \vartheta \partial_{2} \varphi\right)+\frac{1}{4}\left(x_{2}^{4} \cos ^{2} \vartheta+x_{1}^{4} \sin ^{2} \vartheta\right) \varphi=\lambda \varphi \text { in } \mathbb{R}_{+}^{2}, \\
\partial_{2} \varphi+\frac{i}{2} x_{1}^{2} \sin \vartheta \varphi=0 \quad \text { on } \partial \mathbb{R}_{+}^{2} .
\end{array}\right.
$$

The natural space for the operator $-\nabla_{\mathbf{A}}^{2}$ is the magnetic $H^{1}$ space associated with A:

$$
\mathcal{W}^{1,2}\left(\mathbb{R}_{+}^{2}, \mathbf{A}\right)=\left\{\phi \in L^{2}\left(\mathbb{R}_{+}^{2}\right):|\nabla \phi-i \mathbf{A} \phi| \in L^{2}\left(\mathbb{R}_{+}^{2}\right)\right\}
$$


In the following we denote $\lambda\left(\mathbb{R}_{+}^{2}, \mathbf{A}\right)$ by $\lambda\left(\mathbb{R}_{+}^{2}, \vartheta\right)$. Obviously,

$$
\lambda\left(\mathbb{R}_{+}^{2}, \pi+\vartheta\right)=\lambda\left(\mathbb{R}_{+}^{2}, \vartheta\right) .
$$

So we discuss (1.11) for $0 \leq \vartheta<\pi$. The case where $\vartheta=0$ is most interesting. In this case, equation (1.11) reads as follows:

$$
-\Delta \varphi-i x_{2}^{2} \partial_{1} \varphi+\frac{1}{4} x_{2}^{4} \varphi=\lambda \varphi \quad \text { in } \mathbb{R}_{+}^{2}, \quad \partial_{2} \varphi=0 \quad \text { on } \partial \mathbb{R}_{+}^{2} .
$$

We easily see that

$$
\lambda\left(\mathbb{R}_{+}^{2}, 0\right)=\lambda\left(\mathbb{R}^{2}, \mathbf{A}\right)=\lambda_{0}=\lambda\left(\tau_{0}\right) .
$$

Theorem 4. (i) $\lambda\left(\mathbb{R}_{+}^{2}, 0\right)=\lambda_{0}$, and the associated eigenfunctions are $\varphi=$ $c e^{i \tau_{0} x_{1}} u\left(x_{2}\right)$, where $u$ is the positive eigenfunction of (1.9) with $\tau=\tau_{0}$.

(ii) For $0<\vartheta<\pi$ we have $\lambda\left(\mathbb{R}_{+}^{2}, \vartheta\right)<\lambda_{0}$, and the associated eigenfunctions belong to the magnetic $H^{1}$ space $\mathcal{W}^{1,2}\left(\mathbb{R}_{+}^{2}, \mathbf{A}\right)$.

To classify the lowest eigenfunctions of the eigenvalue problems (1.6) and (1.12), we shall use the ideas developed in [LP1], [LP2, [LP5], which was renamed the F-Principle in LP6 (Subsection 1.4). For the reader's convenience we briefly state it here with respect to (1.6). Let $\psi$ be a bounded eigenfunction of (1.6) associated with the lowest eigenvalue $\lambda_{0}$. Formally, we fix $x_{2}$ and denote the Fourier transform of $\varphi$ in the variable $x_{1}$ by $\tilde{\varphi}\left(z, x_{2}\right)=\mathcal{F}\left[\varphi\left(\cdot, x_{2}\right)\right](z)$. Then, for fixed $z, v(t)=\tilde{\varphi}(z, t)$ satisfies an equation for $\lambda=\lambda_{0}$ :

$$
-v^{\prime \prime}+q(t, \tau) v=\lambda v, \quad-\infty<t<+\infty,
$$

where

$$
q(t, \tau)=\frac{1}{4}\left(t^{2}+2 \tau\right)^{2}
$$

We may call (1.13) the F-transformed equation of (1.6) (or F-equation for short). Information on the F-transformed equation (1-dimensional eigenvalue problem) will help us to study the original (2-dimensional) eigenvalue problem. For fixed $\tau$, denote the eigenvalue of (1.13) by $\lambda(\tau)$. Let $\lambda_{0}=\inf _{\tau} \lambda(\tau)$ and assume $\lambda_{0}=\lambda\left(\tau_{0}\right)$. Let $u$ be the eigenfunction of (1.13) associated with the eigenvalue $\lambda_{0}$. We may guess that the least value $\lambda_{0}$ is the eigenvalue of (1.6), and the associated eigenfunctions of (1.6) are given by $\varphi=c e^{i \tau_{0} x_{1}} u\left(x_{2}\right)$. To rigorously verify these results, we follow the main steps in LP2 (proof of Theorem 5.3) and [LP5] (proof of Lemma 3.2).

Step 1. For fixed $\tau$, the lowest eigenvalue of (1.13) is given by

$$
\lambda(\tau)=\inf _{\phi \in \mathcal{W}^{1,2}(\mathbb{R}, q)} \frac{1}{\|\phi\|_{L^{2}(\mathbb{R})}^{2}} \int_{-\infty}^{+\infty}\left\{\left|\phi^{\prime}\right|^{2}+q(t, \tau)|\phi|^{2}\right\} d t,
$$

where

$$
\mathcal{W}^{1,2}(\mathbb{R}, q)=\left\{\phi \in L^{2}(\mathbb{R}): \sqrt{q} \phi \in L^{2}(\mathbb{R})\right\}
$$

We shall show that there exists a unique $\tau_{0}$ such that

$$
\lambda\left(\tau_{0}\right)=\min _{-\infty<\tau<\infty} \lambda(\tau) .
$$

Let $\lambda\left(\mathbb{R}^{2}, \mathbf{A}\right)$ be the lowest eigenvalue of (1.6). Then we can show that $\lambda\left(\mathbb{R}^{2}, \mathbf{A}\right) \leq$ $\lambda\left(\tau_{0}\right)$. 
Step 2. Let $\varphi$ be a bounded eigenfunction of (1.6) associated with the lowest eigenvalue $\lambda\left(\mathbb{R}^{2}, \mathbf{A}\right)$. We can show that there exists $C>0$ such that, for all $a<b$,

$$
\int_{a}^{b} d x_{1} \int_{-\infty}^{+\infty}|\varphi|^{2} d x_{2} \leq C(b-a+1)\|\varphi\|_{L^{\infty}}^{2} .
$$

Let $\tilde{\varphi}\left(z, x_{2}\right)$ be the Fourier transform of $\varphi$ in the sense of distribution. Using the results in Step 1 and (1.15), we can show that, for any $x_{2}, \tilde{\varphi}\left(\cdot, x_{2}\right)$ is supported at the single point $z=\tau_{0}$. Then we conclude that $\varphi\left(x_{1}, x_{2}\right)=e^{i \tau_{0} x_{1}} u\left(x_{2}\right)$, where $u$ is an eigenfunction of the F-transformed equation for $\tau=\tau_{0}$ on $\mathbb{R}$ associated with the lowest eigenvalue $\lambda\left(\tau_{0}\right)$. Moreover, we get $\lambda\left(\mathbb{R}^{2}, \mathbf{A}\right)=\lambda\left(\tau_{0}\right)$.

We can also carry out the same arguments for the eigenvalue problem (1.12) on the half-plane. In this case, the F-equation is

$$
-u^{\prime \prime}+q(t, \tau) u=\lambda u, \quad t>0 ; \quad u^{\prime}(0)=0 .
$$

It is easy to see that the lowest eigenvalue of (1.16) is equal to the lowest eigenvalue of (1.9). So we use the same notation $\lambda(\tau)$ to denote the lowest eigenvalue of (1.16). We have, in addition to (1.14),

$$
\lambda(\tau)=\inf _{\phi \in \mathcal{W}^{1,2}\left(\mathbb{R}_{+}, q\right)} \frac{1}{\|\phi\|_{L^{2}\left(\mathbb{R}_{+}\right)}^{2}} \int_{0}^{+\infty}\left\{\left|\phi^{\prime}\right|^{2}+q(t, \tau)|\phi|^{2}\right\} d t .
$$

Now we see that the crucial part of our study is to prove the uniqueness of minimum points of the lowest eigenvalue $\lambda(\tau)$ of the Sturm-Liouville problem (1.16) associated to $(1.12)$, with $q(t, \tau)=\frac{1}{4}\left(t^{2}+2 \tau\right)^{2}$. This problem is closely related to the parameter variation problem of eigenvalues studied by Dauge and Helffer $[\mathrm{DH}]$. Note that, if $q(t, \tau)$ has the form

$$
q(t, \tau)=q(t+\tau)
$$

then one can use the method in $[\mathrm{DH}]$ to derive the equality

$$
\lambda^{\prime}(\tau)=c(q(\tau)-\lambda(\tau))
$$

which implies that the minimum point $\tau_{0}$ is determined by $\lambda_{0}=\lambda\left(\tau_{0}\right)=q\left(\tau_{0}\right)$. Then, the uniqueness of the minimum points follows if $q(t)$ is a monotone function. Recall that, in order to estimate the upper critical field $H_{C_{3}}$ for large $\kappa$, one is led to the Sturm-Liouville problem with $q(t, \tau)=(t+\tau)^{2}$ (see [LP2], LP4]). From the above discussion we see that $\lambda_{0}=\tau_{0}^{2}$, which implies the uniqueness of the minimum points (see $[\mathrm{DH}],[\mathrm{BH}])^{3}$ However, for the Sturm-Liouville equation (1.16) with $q(t, \tau)=\frac{1}{4}\left(t^{2}+2 \tau\right)^{2}$, which is not in the form (1.18), the uniqueness of minimum points cannot be obtained by using the above method. Instead, we shall combine ODE techniques and variational ideas. We shall show that, if there exist numbers $\tau_{1}<\tau_{2}$ such that $\lambda\left(\tau_{1}\right)=\lambda\left(\tau_{2}\right)=\lambda_{0}$, then we can find a point $\tau$ lying in between $\tau_{1}$ and $\tau_{2}$, and find a number $\lambda<\lambda_{0}$, such that (1.16) has a bounded positive solution for $\tau$ and $\lambda$. This conclusion contradicts the fact that $\lambda_{0}$ is a minimum. To prove this conclusion, we make a change of variables. Let $u$ be a positive solution of (1.16) satisfying $u(0)=1$. We introduce $\phi$ such that

$$
u=\exp \left(-\int_{0}^{t} \phi(s) d s\right)
$$

\footnotetext{
${ }^{3}$ In [LP2] and [LP4], without knowing the results in [DH], a different investigation was carried out, and the uniqueness of the minimum points was proved by analyzing the family of solutions of an associated ordinary differential equation, see [LP2] (Section 7).
} 
Then $\phi$ satisfies the following Riccati type equation:

$$
\phi^{\prime}=f(t, \phi, \tau, \lambda), \quad t>0 ; \quad \phi(0)=0,
$$

where

$$
f(t, \phi, \tau, \lambda)=\phi^{2}+\lambda-q(t, \tau)=\phi^{2}+\lambda-\frac{1}{4}\left(t^{2}+2 \tau\right)^{2} .
$$

We shall prove the conclusion by carefully analyzing the solutions of (1.19).

We organize this paper as follows. In Section 2 we recall the asymptotic behavior at infinity of all positive solutions of an ordinary differential equation. In Section 3 we discuss the Riccati type equation (1.19), study the eigenvalue variation problem for the Sturm-Liouville operator (1.16), and prove the uniqueness of the minimum points of the function $\lambda(\tau)$. In Section 4 we study (1.6) and prove Theorem 3. In Section 5 we study (1.7) and prove Theorem 4 . In Section 6 we study the eigenvalue problem in a bounded smooth 2-dimensional domain and prove Theorem 2 . Then, by using the methods in [LP4] (proofs of Theorem 1.1 and 1.3), Theorem 1 can be proved as a consequence of Theorem 2 .

Acknowledgments. The authors would like to thank Professor B. Helffer for his many valuable comments on the first manuscript. This work was partially supported by the National Natural Science Foundation of China, the Science Foundation of the Ministry of Education of China, the Zhejiang Provincial Natural Science Foundation of China, and NUS Academic Research Grant R-146-000-022-112.

\section{$\S 2$. Preliminaries}

In this section we recall the asymptotic behavior, for a large variable $t$, of positive solutions of the equation

$$
-y^{\prime \prime}+\frac{1}{4}\left(t^{2}+2 \tau\right)^{2} y=\lambda y
$$

The following lemma will be used in our discussion on the Riccati type equation in Section 3.

Lemma 2.1. Fix $\lambda$ and $\tau$.

(i) For any solution $y$ of (2.1) which is positive and bounded near $+\infty$, there exists $c>0$ such that

$$
\lim _{t \rightarrow+\infty} \exp \left(\frac{t^{3}}{6}+\tau t\right) t y(t)=c .
$$

(ii) Moreover, for any $c>0$, (2.1) has exactly one solution $y(t)$ which is defined for large $t$ and satisfies (2.2).

Lemma 2.1 follows from a general result of Sibuya [] (Theorem 6.1), which gives the asymptotic behavior at infinity of a solution of an ordinary differential equation with a polynomial coefficient 4

Remark 2.2. Let $y(t)$ be a bounded solution of (2.1) and $y \not \equiv 0$. Then $y$ does not change its sign for large $t$. Assume $y(t)>0$ for large $t$. Write

$$
y(t)=\exp \left(-\frac{t^{3}}{6}-\tau t\right) w(t)
$$

\footnotetext{
${ }^{4}$ Although it is not stated explicitly in Theorem 6.1 in [S], the uniqueness (up to a scale multiplication) of the positive solutions satisfying (2.2) can be proved by the comparison method.
} 
Then $w$ satisfies the equation

$$
w^{\prime \prime}-\left(t^{2}+2 \tau\right) w^{\prime}+(\lambda-t) w=0, \quad t>0,
$$

and $w^{\prime}(0)=\tau w(0)$. Let $v(t)=t w(t)$. Then $v$ satisfies the equation

$$
v^{\prime \prime}-\left(t^{2}+2 \tau+\frac{2}{t}\right) v^{\prime}+\left(\lambda+\frac{2 \tau}{t}+\frac{2}{t^{2}}\right) v=0 .
$$

Moreover, if either $\lambda>0$, or $\lambda=0$ and $\tau \geq 0$, then $v$ is strictly increasing for large $t$; if either $\lambda<0$, or $\lambda=0$ and $\tau<0$, then $v(t)$ is strictly decreasing for large $t$. In any case, $v(+\infty)$ exists and is a positive number.

\section{§3. Eigenvalue Variation for Sturm-Liouville Operators}

In this section we study the eigenvalue variation problem for the Sturm-Liouville equation

$$
-u^{\prime \prime}+\frac{1}{4}\left(t^{2}+2 \tau\right)^{2} u=\lambda u, \quad t>0 ; \quad u^{\prime}(0)=0 .
$$

Lemma 3.1. For every $\tau$, the lowest eigenvalue of $(1.9)$ on $(-\infty,+\infty)$ is equal to the lowest eigenvalue of (3.1) on $(0,+\infty)$.

Proof. Although the conclusion is obvious, we include a proof here for the reader's convenience. Let us temporarily denote the lowest eigenvalue of $(3.1)$ by $\lambda(\tau)$ and the lowest eigenvalue of (1.9) by $\hat{\lambda}(\tau)$. We shall show that $\hat{\lambda}(\tau)=\lambda(\tau)$.

First we recall that $\hat{\lambda}(\tau)$ is a simple eigenvalue, i.e., it has only one linearly independent eigenfunction. To see this, assume that $w_{1}$ and $w_{2}$ are eigenfunctions of (1.9) associated with $\hat{\lambda}(\tau)$. Then $w_{1}, w_{2} \in L^{2}(\mathbb{R})$. Let $w(t)=w_{1}(0) w_{2}(t)-$ $w_{2}(0) w_{1}(t)$. Then $w$ is also an $L^{2}$ eigenfunction. If $w \neq \equiv 0$, then from the variational characterization of the lowest eigenvalues we know that $w$ does not change its sign. We may assume that $w \geq 0$. Note that $w(0)=0$. So 0 is a minimum point and hence $w^{\prime}(0)=0$. Since $w(0)=w^{\prime}(0)=0$, the uniqueness result for ordinary differential equations shows that $w \equiv 0$. Hence $w_{1}$ and $w_{2}$ are linearly dependent.

Let $u(t)$ be an eigenfunction of (3.1) associated with the lowest eigenvalue $\lambda(\tau)$, and define $y(t)=u(t)$ for $t \geq 0, y(t)=u(-t)$ for $t<0$. Note that $y \in L^{2}(\mathbb{R})$. Using $y(t)$ as a test function, we see that $\hat{\lambda}(\tau) \leq \lambda(\tau)$. Next let $w$ be an eigenfunction of (1.9) associated with the lowest eigenvalue $\hat{\lambda}(\tau)$. We show that $w$ is an even function. Let $\tilde{w}(t)=w(-t)$. Then $\tilde{w}$ is also an eigenfunction of (1.9). Since the lowest eigenvalue is simple, we see that $\tilde{w}=\mathrm{cw}$. Checking this equality at $t=0$, we see that $c=1$, i.e., $w(-t)=\tilde{w}(t)=w(t)$. So $w^{\prime}(0)=0$. Let $u$ be the restriction of $w$ to $[0,+\infty)$. Then $u$ is an eigenfunction of $(3.1)$ with eigenvalue $\hat{\lambda}(\tau)$. So $\lambda(\tau) \leq \hat{\lambda}(\tau)$. Hence they are equal.

In the following we denote by $\lambda(\tau)$ the lowest eigenvalue of $(3.1)$, and let

$$
\lambda_{0}=\inf _{-\infty<\tau<+\infty} \lambda(\tau)
$$

From Lemma 3.1 we see that (1.10) and (3.2) define the same number.

Theorem 3.2. There exists a unique number $\tau_{0}<0$ such that

$$
\lambda\left(\tau_{0}\right)=\lambda_{0}=\min _{-\infty<\tau<+\infty} \lambda(\tau) .
$$


From Lemma 3.1 and results of Montgomery [M] (also see Helffer [H2]), we see that $\lambda_{0}$ is achieved at some $\tau_{0}$, and $\tau_{0}<0$. As mentioned in $\S 1$, to prove Theorem 1, we shall study the Riccati type equation (1.19) associated with (3.1). Fix $\lambda$ and $\tau$. Let $u$ be a positive solution of (3.1) satisfying $u(0)=1$. Set

$$
u(t)=\exp \left(-\int_{0}^{t} \phi(s) d s\right) .
$$

Then $\phi$ satisfies (1.19), where

$$
f(t, \phi, \tau, \lambda)=\phi^{2}+\lambda-\frac{1}{4}\left(t^{2}+2 \tau\right)^{2} .
$$

In particular, for every $\tau$, let $u(\cdot, \tau)$ be the eigenfunction of (3.1) associated with the eigenvalue $\lambda(\tau)$ such that $u(0, \tau)=1$, and let $\phi(\cdot, \tau)$ be the associated solution of (1.19) defined by (3.3). Then $\phi(\cdot, \tau)$ is a global solution of (1.19) with $\lambda=\lambda(\tau)$. Moreover, from Proposition 2.1 we have

$$
\phi(t, \tau) \sim \frac{t^{2}}{2}+\tau-\frac{1}{t} \quad \text { as } t \rightarrow+\infty .
$$

The following lemma gives the structure of the family $\{\phi(\cdot, \tau)\}$.

Lemma 3.3. (i) $g(\tau) \equiv \lambda(\tau)-\tau^{2}$ is strictly increasing in $\tau$.

(ii) If $\tau_{1}<\tau_{2}$, then $\phi\left(t, \tau_{1}\right)<\phi\left(t, \tau_{2}\right)$ for all $t>0$.

Proof. (i) Use the notation $u(t, \tau)$ as above. Let $\tau_{1}<\tau_{2}$. Then

$$
\begin{aligned}
& g\left(\tau_{2}\right)=\lambda\left(\tau_{2}\right)-\tau_{2}^{2}=\frac{\int_{0}^{+\infty}\left\{\left|u^{\prime}\left(t, \tau_{2}\right)\right|^{2}+\left(\frac{t^{4}}{4}+\tau_{2} t^{2}\right) u^{2}\left(t, \tau_{2}\right)\right\} d t}{\int_{0}^{+\infty} u^{2}\left(t, \tau_{2}\right) d t} \\
& >\frac{\int_{0}^{+\infty}\left\{\left|u^{\prime}\left(t, \tau_{2}\right)\right|^{2}+\left(\frac{t^{4}}{4}+\tau_{1} t^{2}\right) u^{2}\left(t, \tau_{2}\right)\right\} d t}{\int_{0}^{+\infty} u^{2}\left(t, \tau_{2}\right) d t} \\
& =\frac{\int_{0}^{+\infty}\left\{\left|u^{\prime}\left(t, \tau_{2}\right)\right|^{2}+\frac{1}{4}\left(t^{2}+2 \tau_{1} t^{2}\right)^{2} u^{2}\left(t, \tau_{2}\right)\right\} d t}{\int_{0}^{+\infty} u^{2}\left(t, \tau_{2}\right) d t}-\tau_{1}^{2} \\
& >\frac{\int_{0}^{+\infty}\left\{\left|u^{\prime}\left(t, \tau_{1}\right)\right|^{2}+\frac{1}{4}\left(t^{2}+2 \tau_{1} t^{2}\right)^{2} u^{2}\left(t, \tau_{1}\right)\right\} d t}{\int_{0}^{+\infty} u^{2}\left(t, \tau_{1}\right) d t}-\tau_{1}^{2}=\lambda\left(\tau_{1}\right)-\tau_{1}^{2}=g\left(\tau_{1}\right) . \\
& \text { (ii) Assume } \tau_{1}<\tau_{2} . \text { Let } T\left(\tau_{1}, \tau_{2}\right)=\sqrt{\frac{g\left(\tau_{2}\right)-g\left(\tau_{1}\right)}{\tau_{2}-\tau_{1}}} . \text { Then, } \\
& \quad f\left(t, \phi, \tau_{1}, \lambda\left(\tau_{1}\right)\right)<f\left(t, \phi, \tau_{2}, \lambda\left(\tau_{2}\right)\right) \quad 0 \leq t<T\left(\tau_{1}, \tau_{2}\right) .
\end{aligned}
$$

Applying the Comparison Principle to (1.19), we conclude that, for $0<t \leq$ $T\left(\tau_{1}, \tau_{2}\right)$,

$$
\phi\left(t, \tau_{1}\right)<\phi\left(t, \tau_{2}\right) .
$$

We shall show that (3.5) is true for all $t>0$. From (3.4) we see that (3.5) holds for sufficiently large $t$. Suppose that (3.5) is not true for all $t$. Then there exists $t_{0}>T\left(\tau_{1}, \tau_{2}\right)$ such that $\phi\left(t_{0}, \tau_{1}\right)=\phi\left(t_{0}, \tau_{2}\right)$ and $\phi^{\prime}\left(t_{0}, \tau_{1}\right) \leq \phi^{\prime}\left(t_{0}, \tau_{2}\right)$, where the prime denotes the derivative in $t$. However, as $t_{0}>T\left(\tau_{1}, \tau_{2}\right)$, we have

$$
\phi^{\prime}\left(t_{0}, \tau_{1}\right)=f\left(t_{0}, \phi\left(t_{0}, \tau_{1}\right), \tau_{1}, \lambda\left(\tau_{1}\right)\right)>f\left(t_{0}, \phi\left(t_{0}, \tau_{2}\right), \tau_{2}, \lambda\left(\tau_{2}\right)\right)=\phi^{\prime}\left(t_{0}, \tau_{2}\right),
$$

a contradiction. Hence (3.5) is true for all $t>0$. 
The following observation will be used later.

Lemma 3.4. Given $\lambda$ and $\tau$, assume that (1.19) has a solution $\phi$ on $[0,+\infty)$ such that

$$
\int_{0}^{+\infty} \phi(t) d t=+\infty
$$

Then $\lambda$ is equal to the lowest eigenvalue $\lambda(\tau)$ of (3.1), and the function u associated with $\phi$ by (3.3) is an eigenfunction.

Proof of Theorem 3.2. Suppose that $\lambda(\tau)$ has at least two minimum points $\tau_{1}<$ $\tau_{2}<0, \lambda\left(\tau_{1}\right)=\lambda\left(\tau_{2}\right)=\lambda_{0}$. We shall show that there exist $\tau^{*} \in\left(\tau_{1}, \tau_{2}\right)$ and $\lambda^{*}<\lambda_{0}$ such that (1.19) has a global solution $\phi$, and

$$
\phi\left(t, \tau_{1}\right)<\phi(t)<\phi\left(t, \tau_{2}\right) \quad \text { for all } t>0 .
$$

Then, using Lemma 3.4, we see that $\lambda^{*}$ is an eigenvalue of (3.1) with $\tau=\tau^{*}$. This contradicts the fact that $\lambda_{0}$ is the minimum of $\lambda(\tau)$.

Step 1. We introduce some notation.

Computation shows that, for $\tau_{1}<\tau<\tau_{2}$,

$$
\begin{array}{lll}
f\left(t, \phi, \tau_{1}, \lambda\left(\tau_{1}\right)\right)<f(t, \phi, \tau, \lambda) & \text { iff } & 0 \leq t<\sqrt{-\frac{\lambda_{0}-\lambda}{\tau-\tau_{1}}-\tau-\tau_{1}}, \\
f(t, \phi, \tau, \lambda)<f\left(t, \phi, \tau_{2}, \lambda\left(\tau_{2}\right)\right) & \text { iff } & 0 \leq t<\sqrt{\frac{\lambda_{0}-\lambda}{\tau_{2}-\tau}-\tau-\tau_{2}} .
\end{array}
$$

Set $\lambda=\lambda^{*}(\tau)=\lambda_{0}-\left(\tau-\tau_{1}\right)\left(\tau_{2}-\tau\right)$. Then

$$
\sqrt{-\frac{\lambda_{0}-\lambda^{*}(\tau)}{\tau-\tau_{1}}-\tau-\tau_{1}}=\sqrt{\frac{\lambda_{0}-\lambda^{*}(\tau)}{\tau_{2}-\tau}-\tau-\tau_{2}}=\sqrt{-\tau_{1}-\tau_{2}} \equiv t^{*} .
$$

So we have, for $\tau_{1}<\tau<\tau_{2}$,

$$
\begin{array}{ll}
f\left(t, \phi, \tau_{1}, \lambda\left(\tau_{1}\right)\right)<f\left(t, \phi, \tau, \lambda^{*}(\tau)\right)<f\left(t, \phi, \tau_{2}, \lambda\left(\tau_{2}\right)\right), & \text { if } \quad 0 \leq t<t^{*}, \\
f\left(t, \phi, \tau_{2}, \lambda\left(\tau_{2}\right)\right)<f\left(t, \phi, \tau, \lambda^{*}(\tau)\right)<f\left(t, \phi, \tau_{1}, \lambda\left(\tau_{1}\right)\right), & \text { if } \quad t>t^{*},
\end{array}
$$

and, for $\tau_{1} \leq \tau<\tau^{\prime} \leq \tau_{2}$,

$$
f\left(t, \phi, \tau, \lambda^{*}(\tau)\right)<f\left(t, \phi, \tau^{\prime}, \lambda^{*}\left(\tau^{\prime}\right)\right) \quad \text { iff } \quad 0 \leq t<t^{*} .
$$

Note that $\lambda^{*}(\tau)<\lambda_{0}$ for $\tau_{1}<\tau<\tau_{2}, \lambda^{*}\left(\tau_{1}\right)=\lambda^{*}\left(\tau_{2}\right)=\lambda_{0}$, and

$$
\min _{\tau_{1} \leq \tau \leq \tau_{2}} \lambda^{*}(\tau)=\lambda^{*}\left(\frac{\tau_{1}+\tau_{2}}{2}\right)=\lambda_{0}-\frac{1}{4}\left(\tau_{2}-\tau_{1}\right)^{2} .
$$

Step 2. Consider local solutions on $\left[0, t^{*}\right]$.

Using (3.7) and applying the Comparison Principle, we see that, for every $\tau_{1}<$ $\tau<\tau_{2}$, there exists a number $t^{*}>0$ such that the equation

$$
\phi^{\prime}=f\left(t, \phi, \tau, \lambda^{*}(\tau)\right)
$$

has a unique solution $\phi^{*}(t, \tau)$ satisfying $\phi(0)=0$, which is well defined on $\left[0, t^{*}\right]$, and

$$
\phi\left(t, \tau_{1}\right)<\phi^{*}(t, \tau)<\phi\left(t, \tau_{2}\right) \quad \text { for } 0<t \leq t^{*} .
$$

Let $a(\tau)=\phi^{*}\left(t^{*}, \tau\right)$. From (3.8) we see that $a(\tau)$ is continuous and strictly increasing for $\tau \in\left[\tau_{1}, \tau_{2}\right]$, and

$$
a\left(\tau_{1}\right)=\phi\left(t^{*}, \tau_{1}\right), \quad a\left(\tau_{2}\right)=\phi\left(t^{*}, \tau_{2}\right) .
$$


Step 3. Consider solutions on $\left[t^{*},+\infty\right)$.

From Lemma 2.1, for every $\tau \in\left(\tau_{1}, \tau_{2}\right)$ and $\lambda=\lambda^{*}(\tau)$, there exists a number $T^{*}(\tau)$ such that $(2.1)$ has a unique solution $y^{*}(t, \tau)$ which is positive for $t>T^{*}(\tau)$, and satisfies

$$
y^{*}(t, \tau) \sim \frac{1}{t} \exp \left(-\frac{t^{3}}{6}-\tau t\right) \quad \text { as } t \rightarrow+\infty .
$$

Let

$$
\psi^{*}(t, \tau)=-\frac{d}{d t} \log y^{*}(t, \tau), \quad t>T^{*}(\tau) .
$$

Then $\psi^{*}(\cdot, \tau)$ solves (3.9) for $t>T^{*}(\tau)$, and for large $t$ it satisfies the following estimate:

$$
\phi\left(t, \tau_{1}\right)<\psi^{*}(t, \tau)<\phi\left(t, \tau_{2}\right) .
$$

Using this and (3.7) and applying the Comparison Principle, we conclude that $\psi^{*}(\cdot, \tau)$ exists on an interval containing $\left[t^{*},+\infty\right)$, and (3.10) holds for all $t \geq t^{*}$. So we can choose $T^{*}(\tau)>t^{*}$.

Let $b(\tau)=\psi^{*}\left(t^{*}, \tau\right)$. From (3.8) and the uniqueness result in Lemma 2.1, we see that $b(\tau)$ is continuous and strictly increasing for $\tau \in\left[\tau_{1}, \tau_{2}\right]$, and

$$
b\left(\tau_{1}\right)=\phi\left(t^{*}, \tau_{1}\right), \quad b\left(\tau_{2}\right)=\phi\left(t^{*}, \tau_{2}\right) .
$$

Step 4. We show that $b(\tau)-a(\tau)$ changes its sign in the interval $\left(\tau_{1}, \tau_{2}\right)$. We prove this by contradiction. Suppose not. We have the following alternatives:

Case 1. $a(\tau)<b(\tau)$ for all $\tau_{1}<\tau<\tau_{2}$;

Case 2. $a(\tau)>b(\tau)$ for all $\tau_{1}<\tau<\tau_{2}$.

Step 4.1. We show that Case 1 cannot happen. Suppose Case 1 happens. Then for every $\tau \in\left(\tau_{1}, \tau_{2}\right)$, the curve of $\phi^{*}(\cdot, \tau)$ lies below the curve of $\psi^{*}(\cdot, \tau)$, and eventually leaves the region bounded by $\phi\left(\cdot, \tau_{1}\right)$ and $\phi\left(\cdot, \tau_{2}\right)$ through the curve $\phi\left(\cdot, \tau_{1}\right)$ at time $t=t(\tau)$, and $t(\tau)>t^{*}$.

Let $[0, T(\tau))$ be the rightward maximal existing interval for $\phi^{*}(t, \tau)$. Then we have $t^{*}<t(\tau)<T(\tau)$, and

$$
\phi^{*}(t, \tau)< \begin{cases}\phi\left(t, \tau_{1}\right) & \text { if } 0<t \leq t^{*} \\ \psi^{*}(t, \tau) & \text { if } t^{*}<t \leq t(\tau) \\ \phi\left(t, \tau_{2}\right) & \text { if } t>t(\tau)\end{cases}
$$

Let

$$
\omega(t)=\phi\left(t, \tau_{2}\right)-\phi^{*}(t, \tau) .
$$

Then $\omega(t)>0$ for $0<t<T(\tau)$, and

$$
\begin{aligned}
\omega^{\prime} & =\left[\phi\left(t, \tau_{2}\right)+\phi^{*}(t, \tau)\right] \omega-\left(\tau_{2}-\tau\right)\left(t^{2}+\tau_{1}+\tau_{2}\right) \\
& <2 \phi\left(t, \tau_{2}\right) \omega-\left(\tau_{2}-\tau\right)\left(t^{2}+\tau_{1}+\tau_{2}\right) .
\end{aligned}
$$

So

$$
0<\omega(t)<\left(\tau_{2}-\tau\right) G(t) \quad \text { for } 0<t<T(\tau)
$$

where

$$
G(t)=-\exp \left(2 \int_{0}^{t} \phi\left(s, \tau_{2}\right) d s\right) \int_{0}^{t}\left(s^{2}+\tau_{1}+\tau_{2}\right) \exp \left(-2 \int_{0}^{s} \phi\left(x, \tau_{2}\right) d x\right) d s .
$$


Since $\phi\left(t, \tau_{2}\right) \sim \frac{t^{2}}{2}+\tau_{2}-\frac{1}{t}$ as $t \rightarrow+\infty$, we see that $\lim _{t \rightarrow+\infty} G(t)=-\infty$, and hence

$$
\sup _{0 \leq t<+\infty} G(t)<+\infty .
$$

Hence $\omega(t)$ remains bounded in its rightward existing interval. This implies that the rightward maximal existing interval of $\phi^{*}(t, \tau)$ is $[0,+\infty)$, i.e., $T(\tau)=+\infty$.

From (3.12) we see that, if $\tau_{2}-\tau$ is small, then $\omega(t)<\frac{\tau_{2}-\tau_{1}}{2}$ for all large $t$. However, from (3.11) we see that, when $t$ is large,

$$
\omega(t)>\phi\left(t, \tau_{2}\right)-\phi\left(t, \tau_{1}\right)=\tau_{2}-\tau_{1}+o(1) .
$$

This contradiction implies that Case 1 cannot happen.

Step 4.2. Similarly we can show that Case 2 cannot happen. To prove this, instead of $\omega(t)$, we consider the function $\xi(t)=\phi^{*}(t, \tau)-\phi\left(t, \tau_{1}\right)$. The details are omitted.

Step 5. From Steps 2-4 we conclude that there exists a number $\tau^{*} \in\left(\tau_{1}, \tau_{2}\right)$ such that $a\left(\tau^{*}\right)=b\left(\tau^{*}\right)$, namely, $\phi^{*}\left(t^{*}, \tau^{*}\right)=\psi^{*}\left(t^{*}, \tau^{*}\right)$. Let

$$
\phi^{*}(t)= \begin{cases}\phi^{*}\left(t, \tau^{*}\right) & \text { if } 0 \leq t \leq t^{*}, \\ \psi^{*}\left(t, \tau^{*}\right) & \text { if } t>t^{*} .\end{cases}
$$

Then $\phi^{*}(t)$ is a solution of (1.19) for $\tau=\tau^{*}, \lambda=\lambda^{*}\left(\tau^{*}\right)$, and

$$
\phi\left(t, \tau_{1}\right)<\phi^{*}(t)<\phi\left(t, \tau_{2}\right) \quad \text { for all } t>0 .
$$

From the asymptotic behavior of $\phi\left(t, \tau_{1}\right)$ we see that (3.6) holds. Thus, from Lemma 3.4 we conclude that when $\tau=\tau^{*}(2.1)$ has an eigenvalue $\lambda^{*}\left(\tau^{*}\right)<\lambda_{0}$. This contradicts the fact that $\lambda_{0}$ is the minimum. Theorem 3.2 is proved.

In the following we always denote by $\tau_{0}$ the (unique) minimum point of the function $\lambda(\tau)$, denote $\lambda_{0}=\lambda\left(\tau_{0}\right)=\min _{\tau} \lambda(\tau)$, and denote by $u$ the positive eigenfunction of (3.1) for $\tau=\tau_{0}$ and $\lambda=\lambda_{0}$.

Proposition 3.5. We have

$$
\begin{aligned}
& \int_{0}^{+\infty} \frac{\partial q}{\partial \tau}\left(t, \tau_{0}\right) u^{2}(t) d t=0 \\
& \int_{0}^{+\infty} \frac{\partial q}{\partial t}\left(t, \tau_{0}\right) u^{2}(t) d t=\left[\lambda_{0}-q\left(0, \tau_{0}\right)\right] u^{2}(0) ; \\
& \int_{0}^{+\infty} t \frac{\partial q}{\partial t}\left(t, \tau_{0}\right) u^{2}(t) d t=2 \int_{0}^{+\infty}\left|u^{\prime}(t)\right|^{2} d t .
\end{aligned}
$$

Proof. For any $h$ we have

$$
\begin{aligned}
\lambda\left(\tau_{0}+h\right) & \leq \frac{\int_{0}^{+\infty}\left\{\left|u^{\prime}\right|^{2}+q\left(t, \tau_{0}+h\right) u^{2}\right\} d t}{\int_{0}^{+\infty} u^{2} d t} \\
& =\lambda\left(\tau_{0}\right)+h \frac{\int_{0}^{+\infty} \frac{\partial q}{\partial \tau}\left(t, \tau_{0}\right) u^{2} d t}{\int_{0}^{+\infty} u^{2} d t}+O\left(h^{2}\right) .
\end{aligned}
$$

So the first equality holds. 
Extend $u$ to negative $t$ by letting $u(t)=u(0)$ for $t<0$. Denote $u_{h}(t)=u(t+h)$ and set

$$
f(h)=\frac{\int_{0}^{+\infty}\left\{\left|u_{h}^{\prime}\right|^{2}+q\left(t, \tau_{0}\right) u_{h}^{2}\right\} d t}{\int_{0}^{+\infty} u_{h}^{2} d t}=\frac{\int_{h}^{+\infty}\left\{\left|u^{\prime}\right|^{2}+q\left(t-h, \tau_{0}\right) u^{2}\right\} d t}{\int_{h}^{+\infty} u^{2} d t} .
$$

$f$ attains its minimum at $h=0$. The second equality follows from $f^{\prime}(0)=0$.

Let $v_{a}(t)=u\left(\frac{t}{a}\right), a>0$, and consider

$$
g(a)=\frac{\int_{0}^{+\infty}\left\{\left|v_{a}^{\prime}\right|^{2}+q\left(t, a \tau_{0}\right) v_{a}^{2}\right\} d t}{\int_{0}^{+\infty} v_{a}^{2} d t} .
$$

$g$ attains its minimum at $a=1$. So $g^{\prime}(1)=0$, which gives the third equality.

Corollary 3.6 $([\mathrm{DH}])$. Assume that $q(t, \tau)=q(t+\tau)$. If $\lambda_{0}$ is the minimum of $\lambda(\tau)$ and $\tau_{0}$ is a minimum point, then $\lambda_{0}=q\left(\tau_{0}\right)$.

Proof. In fact, in this case we have $\frac{\partial q}{\partial \tau}=\frac{\partial q}{\partial t}$. From the first two equalities in Proposition 3.5 we find that $\lambda_{0}=q\left(\tau_{0}\right)$.

For the Sturm-Liouville problem (3.1), $q(t, \tau)=\frac{1}{4}\left(t^{2}+2 \tau\right)^{2}$. Let $u$ be the eigenfunction of (3.1) associated with the minimum of the lowest eigenvalue $\lambda_{0}=$ $\lambda\left(\tau_{0}\right)$. Then we have

$$
\begin{aligned}
& \int_{0}^{+\infty}\left(t^{2}+2 \tau_{0}\right) u^{2}(t) d t=0 \\
& \int_{0}^{+\infty} t\left(t^{2}+2 \tau_{0}\right) u^{2}(t) d t=\left(\lambda_{0}-\tau_{0}^{2}\right) u^{2}(0), \\
& \int_{0}^{+\infty}\left|u^{\prime}(t)\right|^{2} d t=\frac{2 \lambda_{0}}{3} \int_{0}^{+\infty} u^{2}(t) d t \\
& \int_{0}^{+\infty}\left(t^{2}+2 \tau_{0}\right)^{2} u^{2}(t) d t=\frac{4 \lambda_{0}}{3} \int_{0}^{+\infty} u^{2}(t) d t
\end{aligned}
$$

Note that the last two equalities of (3.14) are derived from the first and third equalities in (3.13).

\section{$\S 4$. An Eigenvalue Problem in $\mathbb{R}^{2}$}

In this section we shall prove Theorem 3 concerning the eigenvalue problem (1.6), where

$$
\mathbf{A}=-\frac{1}{2}|x|^{2} \mathbf{n}, \quad \mathbf{n}=(\cos \vartheta, \sin \vartheta) .
$$

We first rotate the coordinate system to simplify (1.6). Set

$$
x_{1}=y_{1} \cos \vartheta-y_{2} \sin \vartheta, \quad x_{2}=y_{1} \sin \vartheta+y_{2} \cos \vartheta
$$

and let

$$
\phi(y)=\psi(x)=\psi\left(y_{1} \cos \vartheta-y_{2} \sin \vartheta, y_{1} \sin \vartheta+y_{2} \cos \vartheta\right) .
$$

Then

$$
\left|\nabla_{\mathbf{A}(x)} \psi(x)\right|^{2}=\left.\left.\left|\frac{\partial \phi}{\partial y_{1}}+\frac{i}{2}\right| y\right|^{2} \phi\right|^{2}+\left|\frac{\partial \phi}{\partial y_{2}}\right|^{2} .
$$


Furthermore, let

$$
\varphi(y)=\exp \left(\frac{i}{6} y_{1}^{3}\right) \phi(y)
$$

We find that

$$
\left.\left.\left|\frac{\partial \phi}{\partial y_{1}}+\frac{i}{2}\right| y\right|^{2} \phi\right|^{2}+\left|\frac{\partial \phi}{\partial y_{2}}\right|^{2}=\left|\frac{\partial \varphi}{\partial y_{1}}+\frac{i}{2} y_{2}^{2} \varphi\right|^{2}+\left|\frac{\partial \varphi}{\partial y_{2}}\right|^{2} .
$$

Thus in the rest of this section we always assume

$$
\mathbf{A}(y)=\left(-\frac{1}{2} y_{2}^{2}, 0\right)
$$

and write (1.6) as follows:

$$
-\Delta \varphi-i y_{2}^{2} \partial_{1} \varphi+\frac{1}{4} y_{2}^{4} \varphi=\lambda \varphi \quad \text { in } \mathbb{R}^{2},
$$

where $\partial_{j}=\frac{\partial}{\partial y_{j}}$. Note that

$$
\operatorname{curl} \mathbf{A}=y_{2} .
$$

Lemma 4.1. For any $C^{1}$ function $\phi$ with compact support in $\mathbb{R}^{2}$,

$$
\int_{\mathbb{R}^{2}}\left|\nabla_{\mathbf{A}} \phi\right|^{2} d x \geq\left.\left|\int_{\mathbb{R}^{2}}(\operatorname{curl} \mathbf{A})\right| \phi\right|^{2} d x \mid .
$$

See $\mathrm{M}$ ] (Theorem 4) for the proof of Lemma 4.1.

As mentioned in $\S 1$, the following lemma is needed in order to apply the Fprinciple.

Lemma 4.2. There exists a constant $C>0$ such that, for any bounded eigenfunction $\varphi$ of (4.2) and for any $a<b$,

$$
\int_{a}^{b} d y_{1} \int_{-\infty}^{+\infty}|\varphi|^{2} d y_{2} \leq C(b-a+1)\|\varphi\|_{L^{\infty}\left(\mathbb{R}^{2}\right)}^{2}
$$

Proof. We follow the line in LP2] (Section 5). Let $\eta$ be a cut-off function with a compact support spt $\eta$. From (4.2) we have

$$
\int_{\mathbb{R}^{2}}\left|\nabla_{\mathbf{A}}(\eta \varphi)\right|^{2} d y=\int_{\mathbb{R}^{2}}\left\{\lambda|\eta \varphi|^{2}+|\nabla \eta|^{2}|\varphi|^{2}\right\} d y
$$

Fix $l>0$ and assume that spt $\eta \subset\left\{\left(y_{1}, y_{2}\right): y_{2}>l\right\}$. From (4.3) we have

$$
\int_{\mathbb{R}^{2}}\left|\nabla_{\mathbf{A}}(\eta \varphi)\right|^{2} d y \geq\left.\left.\left|\int_{\mathbb{R}^{2}} y_{2}\right| \eta \varphi\right|^{2} d y\left|\geq l \int_{\mathbb{R}^{2}}\right| \eta \varphi\right|^{2} d y
$$

Combining this and (4.5), we get

$$
(l-\lambda) \int_{\mathbb{R}^{2}}|\eta \varphi|^{2} d y \leq \int_{\mathbb{R}^{2}}|\nabla \eta|^{2}|\varphi|^{2} d y .
$$

After approximation we may choose $\eta=\eta_{1}\left(y_{1}\right) \eta_{2}\left(y_{2}\right)$, where

$$
\eta_{1}(t)= \begin{cases}e^{\varepsilon(m+t)} & \text { if }-\infty<t<-m, \\ 1 & \text { if }-m \leq t \leq m, \\ e^{\varepsilon(m-t)} & \text { if } \quad m<t<+\infty ;\end{cases}
$$




$$
\eta_{2}(t)= \begin{cases}0 & \text { if }-\infty<t<l, \\ 1 & \text { if } l+1 \leq t \leq l+n, \\ e^{\varepsilon(l+n-t)} & \text { if } l+n<t<+\infty\end{cases}
$$

$0 \leq \eta_{2}(t) \leq 1$ and $\left|\eta^{\prime}(t)\right| \leq 2$ for $l \leq t \leq l+1$. Then we have

$$
\left(l-\lambda-2 \varepsilon^{2}\right) \int_{\mathbb{R}^{2}}|\eta \varphi|^{2} d y \leq 4\|\varphi\|_{L^{\infty}\left(\mathbb{R}_{+}^{2}\right)}^{2} \int_{-\infty}^{+\infty} \eta_{1}^{2} d y_{1} .
$$

Letting $n \rightarrow+\infty$, we get:

$$
\int_{-m}^{m} d y_{1} \int_{0}^{+\infty}|\varphi|^{2} d y \leq\left\{\frac{4\left(2 m+\frac{1}{\varepsilon}\right)}{l-\lambda-2 \varepsilon^{2}}+2 m(l+1)\right\}\|\varphi\|_{L^{\infty}\left(\mathbb{R}_{+}^{2}\right)}^{2} .
$$

Similarly we deal with the integral on $\left\{y_{2}<0\right\}$. Now we fix $l>\lambda+2$ and $0<\varepsilon<1$, and find a constant $C$, depending only on $l$ and $\varepsilon$, such that

$$
\int_{-m}^{m} d y_{1} \int_{0}^{+\infty}|\varphi|^{2} d y \leq C(2 m+1)\|\varphi\|_{L^{\infty}\left(\mathbb{R}_{+}^{2}\right)}^{2} .
$$

(4.4) follows from this inequality by translation.

Proof of Theorem 3. Recall that the lowest eigenvalue of (4.2) is equal to the minimum value $\lambda_{0}$ of the function $\lambda(\tau)$, see $[\mathrm{M}] 5$

Let $\varphi$ be a bounded eigenfunction of (4.2) associated with the lowest eigenvalue $\lambda_{0}$. Let $\tilde{\varphi}\left(z, y_{2}\right)$ be the Fourier transformation of $\varphi\left(y_{1}, y_{2}\right)$ with respect to the variable $y_{1}$ in the sense of distribution. Following the arguments in LP2] (Section $5)$, we can show that, for any $y_{2}$, the support of $\tilde{\varphi}\left(\cdot, y_{2}\right)$ either is empty or contains only the minimum point $\tau_{0}$ of $\lambda(\tau)$. Therefore $\tilde{\varphi}\left(z, y_{2}\right)$ can be represented as

$$
\tilde{\varphi}\left(z, y_{2}\right)=\sum_{k=0}^{N\left(y_{2}\right)} c_{k}\left(y_{2}\right) \frac{d^{k}}{d z^{k}} \delta\left(z-\tau_{0}\right),
$$

where $N\left(y_{2}\right)$ and $c_{k}\left(y_{2}\right)$ may depend on $y_{2}$, and $\delta\left(z-\tau_{0}\right)$ is the Dirac delta function supported at $\tau_{0}$. Since $\varphi$ is bounded, we find that $c_{k}\left(y_{2}\right)=0$ for all $k>0$. Hence

$$
\varphi(y)=u\left(y_{2}\right) \exp \left(i \tau_{0} y_{1}\right)
$$

where $u$ is a bounded function. Plugging it into (4.2), we find that $u\left(y_{2}\right)$ satisfies

$$
-u^{\prime \prime}+\frac{1}{4}\left(y_{2}^{2}+2 \tau_{0}\right)^{2} u=\lambda_{0} u \text {. }
$$

So $u$ is exactly the eigenfunction of (1.9) associated with the lowest eigenvalue $\lambda\left(\tau_{0}\right)=\lambda_{0}$ for $\tau=\tau_{0}$. Theorem 3 is proved.

\section{§5. An Eigenvalue Problem in $\mathbb{R}_{+}^{2}$}

In this section we study the eigenvalue problem (1.7) and prove Theorem 4, where $\mathbf{A}$ was given in Section 1. Let

$$
\varphi(x)=\psi(x) \exp \left(\frac{i x_{1}^{3}}{6} \cos \vartheta+\frac{i x_{2}^{3}}{6} \sin \vartheta\right) .
$$

Then $\varphi$ satisfies (1.11). Hence in the following we assume that

$$
\mathbf{A}(x)=-\left(\frac{x_{2}^{2}}{2} \cos \vartheta, \frac{x_{1}^{2}}{2} \sin \vartheta\right),
$$

\footnotetext{
${ }^{5}$ This conclusion can also be proved by the method in LP2 (Section 5).
} 
and write (1.11) as follows:

$$
-\nabla_{\mathbf{A}}^{2} \psi=\lambda \psi \quad \text { in } \mathbb{R}_{+}^{2}, \quad\left(\nabla_{\mathbf{A}} \psi\right) \cdot \nu=0 \quad \text { on } \partial \mathbb{R}_{+}^{2} .
$$

To simplify notation, in this section we denote $\lambda\left(\mathbb{R}_{+}^{2}, \vartheta\right)$ by $\lambda_{+}(\vartheta)$, namely,

$$
\begin{aligned}
& \lambda_{+}(\vartheta)=\inf _{\phi \in \mathcal{W}^{1,2}\left(\mathbb{R}_{+}^{2}, \mathbf{A}\right)} \frac{\int_{\mathbb{R}_{+}^{2}}\left|\nabla_{\mathbf{A}} \phi\right|^{2} d x}{\int_{\mathbb{R}_{+}^{2}}|\phi|^{2} d x} \\
& =\inf _{\phi \in \mathcal{W}^{1,2}\left(\mathbb{R}_{+}^{2}, \mathbf{A}\right)} \frac{1}{\|\phi\|_{L^{2}\left(\mathbb{R}_{+}^{2}\right)}^{2}} \int_{\mathbb{R}_{+}^{2}}\left\{\left|\left(\partial_{1}+\frac{i}{2} x_{2}^{2} \cos \vartheta\right) \phi\right|^{2}+\left|\left(\partial_{2}+\frac{i}{2} x_{1}^{2} \sin \vartheta\right) \phi\right|^{2}\right\} d x .
\end{aligned}
$$

Lemma 5.1. $\lambda_{+}(\vartheta)=\lambda_{+}(-\vartheta)=\lambda_{+}(\pi+\vartheta)=\lambda_{+}(\pi-\vartheta)$.

Proof. Given a function $\psi$, let $\phi(x)=\psi\left(-x_{1}, x_{2}\right)$. We compute

$$
\begin{aligned}
& \mid\left(\partial_{1}\right.\left.+\frac{i}{2} x_{2}^{2} \cos (\pi-\vartheta)\right)\left.\phi\right|^{2}(x)+\left|\left(\partial_{2}+\frac{i}{2} x_{1}^{2} \sin (\pi-\vartheta)\right) \phi\right|^{2}(x) \\
& \quad=\left|\left(\partial_{1}+\frac{i}{2} x_{2}^{2} \cos \vartheta\right) \psi\right|^{2}\left(-x_{1}, x_{2}\right)+\left|\left(\partial_{2}+\frac{i}{2} x_{1}^{2} \sin \vartheta\right) \psi\right|^{2}\left(-x_{1}, x_{2}\right) .
\end{aligned}
$$

So we have $\lambda_{+}(\pi-\vartheta)=\lambda_{+}(\vartheta)$. Let $\phi(x)=\bar{\psi}\left(-x_{1}, x_{2}\right)$, the complex conjugate of $\psi\left(-x_{1}, x_{2}\right)$. Then,

$$
\begin{aligned}
\mid\left(\partial_{1}\right. & \left.+\frac{i}{2} x_{2}^{2} \cos (-\vartheta)\right)\left.\phi\right|^{2}(x)+\left|\left(\partial_{2}+\frac{i}{2} x_{1}^{2} \sin (-\vartheta)\right) \phi\right|^{2}(x) \\
= & \left|\left(\partial_{1}+\frac{i}{2} x_{2}^{2} \cos \vartheta\right) \psi\right|^{2}\left(-x_{1}, x_{2}\right)+\left|\left(\partial_{2}+\frac{i}{2} x_{1}^{2} \sin \vartheta\right) \psi\right|^{2}\left(-x_{1}, x_{2}\right) .
\end{aligned}
$$

Hence $\lambda_{+}(-\vartheta)=\lambda_{+}(\vartheta)$.

From the above two equalities we get Lemma 5.1.

In the following we assume $0 \leq \vartheta<\pi$.

Lemma 5.2. Let $\lambda_{0}$ be the number given in (1.10).

(i) $\lambda_{+}(0)=\lambda_{0}$, with eigenfunctions given in Theorem 4, conclusion (i).

(ii) $\lambda_{+}(\vartheta)<\lambda_{0}$ if $0<\vartheta<\pi$.

Proof. Step 1. We show (i).

Let $u$ be the eigenfunction of (1.16) with $\tau=\tau_{0}$ associated with the eigenvalue $\lambda_{0}$. Let $\eta_{n}$ be a cut-off function supported in $\left\{\left(x_{1}, x_{2}\right):\left|x_{1}\right|<n\right\}$, and choose

$$
\phi_{n}(x)=\eta_{n}\left(x_{1}\right) e^{i \tau_{0} x_{1}} u\left(x_{2}\right)
$$

as a test function. Computing the related integrals and then letting $n \rightarrow \infty$, we find that $\lambda_{+}(0) \leq \lambda_{0}$.

Note that when $\vartheta=0,(5.1)$ is reduced to (1.12) with the homogeneous Neumann condition on $\partial \mathbb{R}_{+}^{2}$, and $e^{i \tau_{0} x_{1}} u\left(x_{2}\right)$ is a bounded eigenfunction of (5.1) associated with the eigenvalue $\lambda_{0}$. Now, let $\varphi$ be a bounded eigenfunction of (5.1) associated with the lowest eigenvalue $\lambda_{+}(0)$. Then $\partial_{2} \varphi\left(x_{1}, 0\right)=0$. Extend $\varphi$ by letting $\psi(x)=\varphi\left(x_{1}, x_{2}\right)$ for $x_{2} \geq 0$ and $\varphi\left(-x_{1}, x_{2}\right)$ for $x_{2}<0$. Then $\psi$ is an eigenfunction of (1.8). Hence $\lambda_{+}(0) \geq \lambda_{0}$. From Theorem 3 we conclude that $\psi(x)=c e^{i \tau_{0} x_{1}} u\left(x_{2}\right)$. So the conclusion holds.

Step 2. We show that $\lambda_{+}(\vartheta)<\lambda_{0}$ if $0<\vartheta<\pi$ and $\vartheta \neq \frac{\pi}{2}$. From Lemma 5.1 we may assume $0<\vartheta<\frac{\pi}{2}$. We follow the idea in [LP5] (Section 3) to find a test function. 
By gauge invariance, we choose the vector field

$$
\mathbf{A}(x)=-\frac{|x|^{2}}{2}(\cos \vartheta, \sin \vartheta)
$$

Then we introduce new coordinates $y=\left(y_{1}, y_{2}\right)$ as in (4.1). Note that $x_{2}>0$ if and only if $y_{2}>-y_{1} \tan \vartheta$. For any given function $\phi(x)$, we choose $\varphi$ such that

$$
\psi(y)=\phi(x), \quad \varphi(y)=\exp \left(\frac{i y_{1}^{3}}{6}+i \tau_{0} y_{1}\right) \psi(y),
$$

where $\tau_{0}$ is the unique minimum point of $\lambda(\tau)$. Then we have

$$
\left|\nabla_{\mathbf{A}(x)} \phi(x)\right|^{2}=\left.\left.\left|\partial_{y_{1}} \psi+\frac{i}{2}\right| y\right|^{2} \psi\right|^{2}+\left|\partial_{y_{2}} \psi\right|^{2}=\left|\partial_{y_{1}} \varphi+\frac{i}{2}\left(y_{2}^{2}+2 \tau_{0}\right) \varphi\right|^{2}+\left|\partial_{y_{2}} \varphi\right|^{2} .
$$

Now we let $u$ be the positive eigenfunction of (3.1) associated with the lowest eigenvalue $\lambda_{0}=\lambda\left(\tau_{0}\right)$, and choose

$$
\varphi(y)=g\left(-y_{1} \tan \vartheta\right) u\left(y_{2}\right),
$$

where $g$ is a smooth function with compact support and is to be determined later. Let

$$
f(t)=\int_{t}^{+\infty} u^{2}(s) d s
$$

We have

$$
\int_{\mathbb{R}_{+}^{2}}|\varphi|^{2} d y=\int_{-\infty}^{+\infty} d y_{1} \int_{-y_{1} \tan \vartheta}^{+\infty} g^{2}\left(-y_{1} \tan \vartheta\right) u^{2}\left(y_{2}\right) d y_{2}=\cot \vartheta \int_{-\infty}^{+\infty} g^{2}(t) f(t) d t .
$$

Integrating by parts and using (3.1), we get

$$
\begin{aligned}
Q \equiv & \int_{\mathbb{R}_{+}^{2}}\left\{\left|\partial_{y_{1}} \varphi+\frac{i}{2}\left(y_{2}^{2}+2 \tau\right) \varphi\right|^{2}+\left|\partial_{y_{2}} \varphi\right|^{2}\right\} d y \\
= & \tan \vartheta \int_{-\infty}^{+\infty} f(t) g^{\prime}(t)^{2} d t \\
& +\cot \vartheta \int_{-\infty}^{+\infty} g^{2}(t) d t \int_{t}^{+\infty}\left\{\left|u^{\prime}\left(y_{2}\right)\right|^{2}+\frac{1}{4}\left(y_{2}^{2}+2 \tau_{0}\right)^{2} u^{2}\left(y_{2}\right)\right\} d y_{2} \\
= & \lambda_{0} \cot \vartheta \int_{-\infty}^{+\infty} f(t) g^{2}(t) d t+\int_{-\infty}^{+\infty}\left[\tan \vartheta f(t)\left|g^{\prime}(t)\right|^{2}-\cot \vartheta g^{2}(t) u(t) u^{\prime}(t)\right] d t .
\end{aligned}
$$

Noting that $f^{\prime \prime}=-2 u u^{\prime}$, we get

$$
Q-\lambda_{0}\|\varphi\|_{L^{2}\left(\mathbb{R}_{+}^{2}\right)}^{2}=\int_{-\infty}^{+\infty} g^{\prime}(t)\left[\tan \vartheta f(t) g^{\prime}(t)-\cot \vartheta f^{\prime}(t) g(t)\right] d t .
$$

Let $\eta$ be a cut-off function such that $\eta=1$ for $|t| \leq n, \eta=0$ for $|t|>2 n$, and $\left|\eta^{\prime}\right| \leq \frac{2}{n}$. Choose $\varepsilon>0$ small enough so that $\alpha=\cot ^{2} \vartheta-\varepsilon>0$. Let

$$
g=\eta(t) f^{\alpha}(t)
$$


Recall that $u$ decays exponentially. We compute

$$
\begin{aligned}
Q- & \lambda_{0}\|\varphi\|_{L^{2}\left(\mathbb{R}_{+}^{2}\right)}^{2} \\
& =\tan \vartheta \int_{-\infty}^{+\infty}\left\{-\varepsilon \alpha \eta^{2} f^{2 \alpha-1}\left|f^{\prime}\right|^{2}+\left|\eta^{\prime}\right|^{2} f^{2 \alpha+1}+(\alpha-\varepsilon) \eta \eta^{\prime} f^{2 \alpha} f^{\prime}\right\} d t \\
& =-\varepsilon \alpha \tan \vartheta \int_{-\infty}^{+\infty} f^{2 \alpha-1}\left|f^{\prime}\right|^{2} d t+O\left(\frac{1}{n}\right) .
\end{aligned}
$$

Now we choose large $n$ such that

$$
Q-\lambda_{0}\|\varphi\|_{L^{2}\left(\mathbb{R}_{+}^{2}\right)}^{2}<0 .
$$

Returning to the original variables, we find a test function, and conclude that $\lambda_{+}(\vartheta)<\lambda_{0}$.

Step 3. We show that $\lambda_{+}\left(\frac{\pi}{2}\right)<\lambda_{0}$.

Let $u$ be the positive eigenfunction of the equation (1.9) associated with the lowest eigenvalue $\lambda_{0}=\lambda\left(\tau_{0}\right)$ such that $\|u\|_{L^{2}}=1$. Choose

$$
w=u\left(x_{1}\right) g\left(x_{2}\right), \quad \chi=a\left(x_{1}^{2}+2 \tau_{0}\right) \xi\left(x_{2}\right), \quad \phi=w \exp \left(i \tau_{0} x_{2}+i \chi\right),
$$

where $a>0, g$ and $\xi$ are real functions, all to be determined later. We have

$$
\begin{aligned}
& Q \equiv \int_{\mathbb{R}_{+}^{2}}\left\{\left|\partial_{1} \phi\right|^{2}+\left|\partial_{2} \phi+\frac{i}{2} x_{1}^{2} \phi\right|^{2}\right\} d x \\
& =\int_{\mathbb{R}_{+}^{2}}\left\{|\nabla u|^{2}+u^{2}\left[|\nabla \chi|^{2}+\left(x_{1}^{2}+2 \tau_{0}\right) \partial_{2} \chi+\frac{1}{4}\left(x_{1}^{2}+2 \tau_{0}\right)^{2}\right]\right\} d x=\lambda_{0}\|\phi\|_{L^{2}\left(\mathbb{R}_{+}^{2}\right)}^{2}+I
\end{aligned}
$$

where

$$
\begin{aligned}
I= & \int_{-\infty}^{+\infty} u^{2}\left(x_{1}\right) d x_{1} \int_{0}^{+\infty}\left\{\left|g^{\prime}\left(x_{2}\right)\right|^{2}+g^{2}\left(x_{2}\right)\left[|\nabla \chi|^{2}+\left(x_{1}^{2}+2 \tau_{0}\right) \partial_{2} \chi\right]\right\} d x_{2} \\
= & \int_{-\infty}^{+\infty} u^{2}\left(x_{1}\right) d x_{1} \int_{0}^{+\infty}\left\{\left|g^{\prime}\left(x_{2}\right)\right|^{2}\right. \\
& \left.\quad+g^{2}\left(x_{2}\right)\left[4 a^{2} x_{1}^{2} \xi^{2}\left(x_{2}\right)+a^{2}\left(x_{1}^{2}+2 \tau_{0}\right)^{2}\left|\xi^{\prime}\left(x_{2}\right)\right|^{2}+a\left(x_{1}^{2}+2 \tau_{0}\right)^{2} \xi^{\prime}\left(x_{2}\right)\right]\right\} d x_{2} \\
= & \int_{0}^{+\infty}\left\{\left|g^{\prime}\left(x_{2}\right)\right|^{2}+g^{2}\left(x_{2}\right)\left[c_{1} a^{2} x_{1}^{2} \xi^{2}\left(x_{2}\right)+c_{2} a^{2}\left|\xi^{\prime}\left(x_{2}\right)\right|^{2}+c_{2} a \xi^{\prime}\left(x_{2}\right)\right]\right\} d x_{2} .
\end{aligned}
$$

Here

$$
\begin{aligned}
& c_{1}=4 \int_{-\infty}^{+\infty} x_{1}^{2} u^{2}\left(x_{1}\right) d x_{1}, \\
& c_{2}=\int_{-\infty}^{+\infty}\left(x_{1}^{2}+2 \tau_{0}\right)^{2} u^{2}\left(x_{1}\right) d x_{1}=\frac{4 \lambda_{0}}{3} .
\end{aligned}
$$

Here we have used the fact $\|u\|_{L^{2}}=1$. Now we fix $b>0$. Choose $l>0$ large, $\mu>0$ and $\varepsilon>0$ small, such that

$$
\mu\left(b^{2}+\frac{c_{1}}{c_{2}}\right)<\frac{1}{2}, \quad e^{-b l}<\frac{1}{2}, \quad 0<\varepsilon<\frac{c_{2} \mu b}{4} .
$$

Let

$$
a=\mu b, \quad \xi\left(x_{2}\right)=e^{-b x_{2}}
$$


and (after approximation)

$$
g(t)= \begin{cases}1 & \text { if } 0 \leq t \leq l, \\ e^{-\varepsilon(t-l)} & \text { if } l<t<+\infty .\end{cases}
$$

We have

$$
\begin{aligned}
& c_{1} a^{2} x_{1}^{2} \xi^{2}\left(x_{2}\right)+c_{2} a^{2}\left|\xi^{\prime}\left(x_{2}\right)\right|^{2}+c_{2} a \xi^{\prime}\left(x_{2}\right) \\
& =-c_{2} a b e^{-b x_{2}}\left(1-a b e^{-b x_{2}}-\frac{a c_{1}}{b c_{2}} e^{-b x_{2}}\right) \leq \frac{c_{2} \mu b^{2}}{2} e^{-b x_{2}} .
\end{aligned}
$$

Then

$$
\begin{aligned}
I & \leq \int_{0}^{+\infty}\left\{\left|g^{\prime}\right|^{2}\left(x_{2}\right)-\frac{c_{2} \mu b^{2}}{2} e^{-b x_{2}} g^{2}\left(x_{2}\right)\right\} d x_{2} \\
& <\frac{\varepsilon}{2}-\frac{c_{2} \mu b^{2}}{2}\left(1-e^{-b l}\right)<\frac{\varepsilon}{2}-\frac{c_{2} \mu b}{4}<-\frac{c_{2} \mu b}{8}<0 .
\end{aligned}
$$

With the above choice we have $Q-\lambda_{0}\|\phi\|_{L^{2}\left(\mathbb{R}_{+}^{2}\right)}^{2}<0$. So $\lambda_{+}\left(\frac{\pi}{2}\right)<\lambda_{0}$.

Lemma 5.3. $\lambda_{+}(\vartheta)$ has eigenfunctions in $\mathcal{W}^{1,2}\left(\mathbb{R}_{+}^{2}, \mathbf{A}\right)$ when $0<\vartheta<\pi$.

Proof. Note that if $\varphi$ is an eigenfunction of $\lambda_{+}(\vartheta)$ for $0<\vartheta<\frac{\pi}{2}$, then $\varphi\left(-x_{1}, x_{2}\right)$ is an eigenfunction of $\lambda_{+}(\pi-\vartheta)$. So we only need to consider $\vartheta$ that lies in $\left(0, \frac{\pi}{2}\right]$.

Step 1. In order to prove the existence of eigenfunctions in $\mathcal{W}^{1,2}\left(\mathbb{R}_{+}^{2}, \mathbf{A}\right)$, we consider a series of approximation problems. Define

$$
\begin{aligned}
B_{n}^{+} & =\left\{x \in \mathbb{R}_{+}^{2}:|x|<n\right\}, \\
\mathcal{W}_{n} & =\left\{\phi \in \mathcal{W}^{1,2}\left(B_{n}^{+}, \mathbf{A}\right): \phi(x)=0 \text { when }|x|=n\right\}, \\
\lambda_{n}(\vartheta) & =\inf _{\phi \in \mathcal{W}_{n}} \frac{\int_{B_{n}^{+}}\left|\nabla_{\mathbf{A}} \phi\right|^{2} d x}{\int_{B_{n}^{+}}|\phi|^{2} d x} .
\end{aligned}
$$

It is easy to see that $\lambda_{n}(\vartheta)$ is achieved. Obviously $\left\{\lambda_{n}(\vartheta)\right\}_{n=1}^{\infty}$ is a decreasing sequence, and $\lim _{n \rightarrow \infty} \lambda_{n}(\vartheta)=\lambda_{+}(\vartheta)$. Let $\psi_{n}$ be a minimizer for $\lambda_{n}(\vartheta)$ such that $\left\|\psi_{n}\right\|_{L^{2}\left(B_{n}^{+}\right)}=1$. Then $\psi_{n}$ satisfies

$$
\begin{cases}-\nabla_{\mathbf{A}}^{2} \psi_{n}=\lambda_{n}(\vartheta) \psi_{n} & \text { in } B_{n}^{+}, \\ \left(\nabla_{\mathbf{A}} \psi_{n}\right) \cdot \nu=0 & \text { if } x_{2}=0, \\ \psi_{n}=0 & \text { if }|x|=n .\end{cases}
$$

The elliptic estimates show that $\left\{\psi_{n}\right\}$ is uniformly bounded. Now we extend $\psi_{n}$ onto $\mathbb{R}_{+}^{2}$ by letting $\psi_{n}=0$ on $\overline{\mathbb{R}_{+}^{2}} \backslash B_{n}^{+}$. We have

$$
\int_{\mathbb{R}_{+}^{2}}\left|\nabla_{\mathbf{A}} \psi_{n}\right|^{2} d x=\lambda_{n}(\vartheta) \int_{\mathbb{R}_{+}^{2}}\left|\psi_{n}\right|^{2} d x=\lambda_{n}(\vartheta) .
$$

Step 2. In the following we show that $\left\{\psi_{n}\right\}$ has a subsequence that strongly converges in $L^{2}\left(\mathbb{R}_{+}^{2}\right)$.

Claim 1. For any $\delta>0$ there exists $C=C(\delta)>0$ such that, for all $x \in B_{n}^{+}$and all $n$,

$$
\left|\psi_{n}(x)\right| \leq C\left\|\psi_{n}\right\|_{L^{\infty}} \exp \left(-\delta\left|x_{2} \cos \vartheta-x_{1} \sin \vartheta\right|\right)
$$


To prove (5.4), we introduce new coordinates $y=\left(y_{1}, y_{2}\right)$ by (4.1) and let $\tilde{\psi}_{n}(y)=\psi_{n}(x)$. Let

$$
\begin{aligned}
& \Omega_{n}=\left\{y \in \mathbb{R}_{+}^{2}:|y|<n, y_{1} \sin \vartheta+y_{2} \cos \vartheta>0\right\}, \\
& \Gamma_{n}=\left\{y \in \partial \Omega_{n}: y_{1} \sin \vartheta+y_{2} \cos \vartheta=0\right\}, \\
& S_{n}=\left\{y \in \partial \Omega_{n}:|y|=n\right\} .
\end{aligned}
$$

Then $\tilde{\psi}_{n}$ satisfies the following equation:

$$
\begin{cases}\Delta \tilde{\psi}_{n}+i y_{2}^{2} \partial_{y_{1}} \tilde{\psi}_{n}-\frac{y_{2}^{4}}{4} \tilde{\psi}_{n}+\lambda_{n}(\vartheta) \tilde{\psi}_{n}=0 & \text { in } \Omega_{n}, \\ \frac{\partial \tilde{\psi}_{n}}{\partial \nu}+\frac{i}{2} y_{2}^{2} \nu_{1} \tilde{\psi}_{n}=0 & \text { on } \Gamma_{n} \\ \tilde{\psi}_{n}=0 & \text { on } S_{n} .\end{cases}
$$

Here $\nu=\left(\nu_{1}, \nu_{2}\right)$ is the unit outward normal vector of $\Gamma_{n}$.

Introduce

$$
\phi_{n}(y)=e^{\delta\left|y_{2}\right|} \tilde{\psi}_{n}(y) .
$$

We shall show that $\left\{\phi_{n}\right\}$ is uniformly bounded. Then (5.4) follows.

Suppose it were not true. After passing to a subsequence we may assume that for every $n$ there exists a point $\left(a_{n}, b_{n}\right) \in \Omega_{n}$ such that

$$
\left|\phi_{n}\left(a_{n}, b_{n}\right)\right|=\left\|\phi_{n}\right\|_{L^{\infty}\left(\Omega_{n}\right)} \rightarrow \infty \quad \text { as } n \rightarrow \infty .
$$

Recall that $\left\{\psi_{n}\right\}$ is uniformly bounded. So we must have

$$
\lim _{n \rightarrow \infty}\left|b_{n}\right|=\infty .
$$

In the following we shall consider the rescaled functions near $\left(a_{n}, b_{n}\right)$. Without loss of generality we may assume $b_{n} \rightarrow+\infty$, and rewrite the equation in the region

$$
\Omega_{n}^{+}=\left\{y \in \Omega_{n}: y_{2}>0\right\}
$$

(if $b_{n} \rightarrow-\infty$ we rewrite the equation in the region $\Omega_{n}^{-}=\left\{y \in \Omega_{n}: y_{2}<0\right\}$ ). In the region $\Omega_{n}^{+}$we have $\phi_{n}(y)=e^{\delta y_{2}} \tilde{\psi}_{n}(y)$, and we write the equation for $\phi_{n}$ as follows:

$$
\begin{cases}\Delta \phi_{n}+i y_{2}^{2} \partial_{y_{1}} \phi_{n}-2 \delta \partial_{y_{2}} \phi_{n}-\frac{y_{2}^{4}}{4} \phi_{n}+\left(\lambda_{n}(\vartheta)+\delta^{2}\right) \phi_{n}=0 & \text { in } \Omega_{n} \\ \frac{\partial \phi_{n}}{\partial \nu}+\frac{i}{2} y_{2}^{2} \nu_{1} \phi_{n}-\delta \nu_{2} \phi_{n}=0 & \text { on } \Gamma_{n} \\ \phi_{n}=0 & \text { on } S_{n} .\end{cases}
$$

Let

$$
\varepsilon_{n}=\frac{1}{\sqrt{b_{n}}}, \quad \varphi_{n}(z)=\frac{1}{\left\|\phi_{n}\right\|_{L^{\infty}}} \exp \left(\frac{i}{2} \varepsilon_{n} b_{n}^{2} z_{1}\right) \phi_{n}\left(a_{n}+\varepsilon_{n} z_{1}, b_{n}+\varepsilon_{n} z_{2}\right) .
$$

Then $\varphi_{n}(z)$ satisfies the following equation:

$$
\begin{cases}\Delta \varphi_{n}+i\left(2 z_{2}+\varepsilon_{n}^{3} z_{2}^{2}\right) \partial_{z_{1}} \varphi_{n}-2 \delta \varepsilon_{n} \partial_{z_{2}} \varphi_{n} & \\ =\left[\left(z_{2}+\frac{1}{2} \varepsilon_{n}^{3} z_{2}^{2}\right)^{2}-\varepsilon_{n}^{2}\left(\lambda_{n}(\vartheta)+\delta^{2}\right)\right] \varphi_{n} & \text { in } \tilde{\Omega}_{n} \\ \frac{\partial \varphi_{n}}{\partial \nu}+i\left(z_{2}+\frac{1}{2} \varepsilon_{n}^{3} z_{2}^{2}\right) \nu_{1} \varphi_{n}-\varepsilon_{n} \delta \nu_{2} \varphi_{n}=0 & \text { on } \tilde{\Gamma}_{n} \\ \varphi_{n}=0 & \text { on } \tilde{S}_{n}\end{cases}
$$


where

$$
\tilde{\Omega}_{n}=\frac{\Omega_{n}-\left(a_{n}, b_{n}\right)}{\varepsilon_{n}}, \quad \tilde{\Gamma}_{n}=\frac{\Gamma_{n}-\left(a_{n}, b_{n}\right)}{\varepsilon_{n}}, \quad \tilde{S}_{n}=\frac{S_{n}-\left(a_{n}, b_{n}\right)}{\varepsilon_{n}} .
$$

Note that $\left|\varphi_{n}(0)\right|=1=\left\|\varphi_{n}\right\|_{L^{\infty}}$.

After passing to a subsequence, we have the following cases to deal with.

Case 1. $\lim _{n \rightarrow \infty} \frac{1}{\varepsilon_{n}} \operatorname{dist}\left(\left(a_{n}, b_{n}\right), \partial \Omega_{n}\right)=+\infty$.

Case 2. $\frac{1}{\varepsilon_{n}} \operatorname{dist}\left(\left(a_{n}, b_{n}\right), \Gamma_{n}\right)$ is bounded, $\lim _{n \rightarrow \infty} \frac{1}{\varepsilon_{n}} \operatorname{dist}\left(\left(a_{n}, b_{n}\right), S_{n}\right)=+\infty$.

Case 3. $\lim _{n \rightarrow \infty} \frac{1}{\varepsilon_{n}} \operatorname{dist}\left(\left(a_{n}, b_{n}\right), \Gamma_{n}\right)=+\infty, \frac{1}{\varepsilon_{n}} \operatorname{dist}\left(\left(a_{n}, b_{n}\right), S_{n}\right)$ is bounded.

Case 4. Both $\frac{1}{\varepsilon_{n}} \operatorname{dist}\left(\left(a_{n}, b_{n}\right), \Gamma_{n}\right)$ and $\frac{1}{\varepsilon_{n}} \operatorname{dist}\left(\left(a_{n}, b_{n}\right), S_{n}\right)$ are bounded.

Applying the elliptic estimates to the equation (5.5), we conclude that $\left\{\varphi_{n}\right\}$ is uniformly bounded in $C_{l o c}^{2+\alpha}$. Therefore we can find a subsequence of $\left\{\varphi_{n}\right\}$ which converges in $C_{l o c}^{2+\alpha}$ to a function $\varphi(z)$ with $|\varphi(0)|=1$, and $\varphi$ is a solution of the equation $\nabla_{\mathbf{E}}^{2} \varphi=0$ in $\Omega_{\infty}$, namely

$$
\Delta \varphi+2 i z_{2} \partial_{z_{1}} \varphi-z_{2}^{2} \varphi=0 \quad \text { in } \Omega_{\infty},
$$

where $\mathbf{E}=\left(-z_{2}, 0\right)$, and $\Omega_{\infty}$ is the limit of $\left\{\tilde{\Omega}_{n}\right\}$. After rotation and translation of the coordinate system and making a gauge transform if necessary, we may identify $\Omega_{\infty}$ as follows.

In case $1, \Omega_{\infty}=\mathbb{R}^{2}$. Recall that the lowest eigenvalue of $-\nabla_{\mathbf{E}}^{2}$ in $\mathbb{R}^{2}$ is 1 ; see LP2] (Theorem 1).

In case $2, \Omega_{\infty}=\mathbb{R}_{+}^{2}$. The boundary condition is $\left(\nabla_{\mathbf{E}} \varphi\right) \cdot \nu=0$ on $\partial \mathbb{R}_{+}^{2}$. Recall that the lowest eigenvalue of $-\nabla_{\mathbf{E}}^{2}$ in $\mathbb{R}_{+}^{2}$ under this boundary condition is $\beta_{0}>0$; see [LP2] (Theorem 2).

In case $3, \Omega_{\infty}=\mathbb{R}_{+}^{2}$. The boundary condition is $\varphi=0$ on $\partial \mathbb{R}_{+}^{2}$. Recall that the lowest eigenvalue of $-\nabla_{\mathbf{E}}^{2}$ in $\mathbb{R}_{+}^{2}$ under the Dirichlet boundary condition is 1 ; see [LP2] (Theorem 4).

In case $4, \Omega_{\infty}=\mathbb{Q}=\left\{z=\left(z_{1}, z_{2}\right): z_{1}>0, z_{2}>0\right\}$. The boundary condition is

$$
\left(\nabla_{\mathbf{E}} \varphi\right) \cdot \nu=0 \quad \text { if } z_{1}>0, z_{2}=0, \quad \varphi=0 \quad \text { if } z_{1}=0, z_{2}>0 .
$$

It is easy to see (by odd extension in the $z_{1}$ direction) that the lowest eigenvalue of $-\nabla_{\mathbf{E}}^{2}$ in $\mathbb{Q}$ under these boundary conditions is greater than $\beta_{0}$.

Hence, in each case (5.6) has no non-trivial bounded solutions. Thus none of the above cases can happen. (5.4) is proved.

Step 3. From (5.4) we see that, for any fixed $l>0$ and for any $\varepsilon>0$, there exists $m=m(\varepsilon)$ such that, for all $n$, the solution $\psi_{n}$ of (5.3) given above satisfies

$$
\int_{\left\{\left|x_{1}\right| \geq m, 0 \leq x_{2} \leq l\right\}}\left|\psi_{n}\right|^{2} d x<\varepsilon .
$$

Claim 2. For any $\varepsilon>0$, there exist positive numbers $N$ and $l$ such that, for all $n>N$,

$$
\int_{-\infty}^{+\infty} d x_{1} \int_{l}^{+\infty}\left|\psi_{n}\right|^{2} d x_{2}<\varepsilon
$$


Let $\eta$ be a smooth cut-off function. Multiplying (5.3) by $\eta^{2} \bar{\psi}_{n}$ and integrating, since $\psi_{n}=0$ on $\mathbb{R}_{+}^{2} \backslash B_{n}^{+}$, we get

$$
\int_{\mathbb{R}_{+}^{2}}\left|\nabla_{\mathbf{A}}\left(\eta \psi_{n}\right)\right|^{2} d x=\int_{\mathbb{R}_{+}^{2}}\left\{\lambda_{n}(\vartheta)\left|\eta \psi_{n}\right|^{2}+|\nabla \eta|^{2}\left|\psi_{n}\right|^{2}\right\} d x
$$

Recall that $\lambda_{n}(\vartheta) \rightarrow \lambda_{+}(\vartheta)<\lambda_{0}$ as $n \rightarrow \infty$. Fix $\delta>0$ small enough so that $2 \delta^{2}<\lambda_{0}-\lambda_{n}(\vartheta)$ for all large $n$. Let $\eta(x)=\eta_{1}\left(x_{1}\right) \eta_{2}\left(x_{2}\right)$, where $\eta_{2}(0)=0$ and

$$
\eta_{1}\left(x_{1}\right)=\left\{\begin{array}{llc}
e^{\delta\left(m+x_{1}\right)} & \text { if } & x_{1}<-m, \\
1 & \text { if } & \left|x_{1}\right| \leq m \\
e^{\delta\left(m-x_{1}\right)} & \text { if } & x_{1}>m .
\end{array}\right.
$$

Since spt $\left(\eta \psi_{n}\right) \subset \mathbb{R}_{+}^{2}$, we have

$$
\int_{\mathbb{R}_{+}^{2}}\left|\nabla_{\mathbf{A}}\left(\eta \psi_{n}\right)\right|^{2} d x=\int_{\mathbb{R}^{2}}\left|\nabla_{\mathbf{A}}\left(\eta \psi_{n}\right)\right|^{2} d x \geq \lambda_{0} \int_{\mathbb{R}^{2}}\left|\eta \psi_{n}\right|^{2} d x
$$

Combining this with (5.9), we find that

$$
\left(\lambda_{0}-\lambda_{n}(\vartheta)-\delta^{2}\right) \int_{\mathbb{R}_{+}^{2}} \eta_{1}^{2} \eta_{2}^{2}\left|\psi_{n}\right|^{2} d x \leq \int_{\mathbb{R}_{+}^{2}} \eta_{1}^{2}\left|\eta_{2}^{\prime}\right|^{2}\left|\psi_{n}\right|^{2} d x
$$

Letting $m \rightarrow+\infty$, we get

$$
\left(\lambda_{0}-\lambda_{n}(\vartheta)-\delta^{2}\right) \int_{\mathbb{R}_{+}^{2}} \eta_{2}^{2}\left|\psi_{n}\right|^{2} d x \leq \int_{\mathbb{R}_{+}^{2}}\left|\eta_{2}^{\prime}\right|^{2}\left|\psi_{n}\right|^{2} d x .
$$

Choose $\eta_{2}$ such that $\eta_{2}\left(x_{2}\right)=0$ if $x_{2}<\frac{l}{2}$ and $\eta_{2}\left(x_{2}\right)=1$ if $x_{2}>l$. From the above inequality we get

$$
\left(\lambda_{0}-\lambda_{n}(\vartheta)-\delta^{2}\right) \int_{-\infty}^{+\infty} d x_{1} \int_{l}^{+\infty}\left|\psi_{n}\right|^{2} d x_{2} \leq \frac{C}{l^{2}} .
$$

So (5.8) is true.

Step 4. From (5.7) and (5.8) we see that the sequence $\left\{\psi_{n}\right\}$ is precompact in $L^{2}\left(\mathbb{R}_{+}^{2}\right)$. After passing to a subsequence we may assume that $\psi_{n} \rightarrow \psi$ weakly in $W^{1,2}\left(\mathbb{R}_{+}^{2}\right)$ and strongly in $L^{2}\left(\mathbb{R}_{+}^{2}\right)$. Then $\psi \in \mathcal{W}^{1,2}\left(\mathbb{R}_{+}^{2}, \mathbf{A}\right)$ is an eigenfunction of $\lambda_{+}(\vartheta)$.

Proof of Theorem 4. Theorem 4 is a consequence of Lemmas 5.2 and 5.3.

\section{§6. An Eigenvalue Problem in Bounded Domains}

Let $\Omega$ be a smooth bounded and simply-connected domain in $\mathbb{R}^{2}$ and $\mathbf{F}$ be a smooth vector field. Denote by $\mu=\mu(b \mathbf{F})$ the lowest eigenvalue of the equation (1.5) in $\Omega$. Then

$$
\mu(b \mathbf{F})=\inf _{\phi \in W^{1,2}(\Omega)} \frac{\int_{\Omega}\left|\nabla_{b \mathbf{F}} \phi\right|^{2} d x+\gamma \int_{\partial \Omega}|\phi|^{2} d s}{\int_{\Omega}|\phi|^{2} d x} .
$$

Proof of Theorem 2. We shall estimate the value of $\mu(b \mathbf{F})$ for large $b$, where curl $\mathbf{F}$ has non-degenerate zeros, namely, $H_{0}=$ curl $\mathbf{F}$ satisfies the condition (1.1) stated in the Introduction. We shall adopt the arguments in [LP3] (Sections 6 and 7). So only the outline is given below. 
To derive the upper bound for $\mu(b \mathbf{F})$, we choose a test function by modifying the eigenfunctions of (1.6) and (1.11), and use the local decomposition formula in [LP3] (Lemmas 3.1, 3.2). We can show that

$$
\limsup _{b \rightarrow \infty} \frac{\mu(b \mathbf{F})}{|b|^{2 / 3}} \leq \alpha_{1}\left(H_{0}\right)^{2 / 3},
$$

where $\alpha_{1}\left(H_{0}\right)$ was given in (1.3).

To derive a lower bound, let $b \rightarrow+\infty$ and let $\psi_{b}(x)$ be the eigenfunction of (1.5) such that $\left\|\psi_{b}\right\|_{L^{\infty}(\Omega)}=1$. Let $x^{b}$ be the maximum point of $\left|\psi_{b}\right|$. After passing to a subsequence we may assume that $x^{b} \rightarrow x^{0}$ as $b \rightarrow+\infty$. First, assume $x^{0} \in \Omega$. For simplicity of notation we assume that $x^{0}=0$. From the local decomposition formula given in [LP3] (Lemma 3.1) we have

$$
\mathbf{F}(x)=\nabla \chi+\operatorname{curl} \mathbf{F}(0) \omega(x)-\frac{1}{2}|x|^{2} \operatorname{curl}^{2} \mathbf{F}(0)+\mathbf{D}(x),
$$

where $\chi$ is a smooth function, $\omega(x)=\left(-\frac{x_{2}}{2}, \frac{x_{1}}{2}\right)$ and $|\mathbf{D}(x)| \leq C|x|^{3}$. From our assumption we have curl $\mathbf{F}(0)=0, \operatorname{curl}^{2} \mathbf{F}(0) \neq 0$. Denote

$$
\mathbf{n}=\frac{\operatorname{curl}^{2} \mathbf{F}(0)}{\left|\operatorname{curl}^{2} \mathbf{F}(0)\right|}=(\cos \vartheta, \sin \vartheta), \quad \rho=\left|\operatorname{curl}^{2} \mathbf{F}(0)\right|=\left|\nabla H_{0}(0)\right| .
$$

By gauge invariance we may assume $\chi=0$. So we write

$$
\mathbf{F}(x)=-\frac{\rho|x|^{2}}{2} \mathbf{n}+\mathbf{D}(x) \text {. }
$$

Set

$$
\begin{aligned}
& \varepsilon=\frac{1}{(b \rho)^{1 / 3}}, \quad \Omega_{\varepsilon}=\frac{\Omega-x^{0}}{\varepsilon}=\frac{\Omega}{\varepsilon}, \quad \varphi_{\varepsilon}(y)=\psi_{b}(\varepsilon y), \\
& \mathbf{A}(y)=-\frac{|y|^{2}}{2} \mathbf{n}, \quad \mathbf{D}_{\varepsilon}(y)=\frac{\mathbf{D}(\varepsilon y)}{\varepsilon^{2}} .
\end{aligned}
$$

From (1.5) we find that

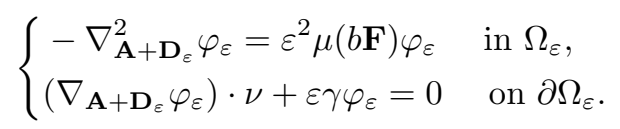

As in [LP3] (Section 7), we can show that $\left\{\varphi_{\varepsilon}\right\}$ has a subsequence $\left\{\varphi_{\varepsilon_{j}}\right\}$ which converges in $C_{l o c}^{2+\alpha}$ to a function $\varphi$, and $\varphi$ satisfies (1.6) in $\mathbb{R}^{2}$ with

$$
\lambda=\lim _{j \rightarrow \infty} \varepsilon_{j}^{2} \mu\left(b_{j} \mathbf{F}\right) .
$$

From Theorem 3, if $\varphi \not \equiv 0$ we must have $\lambda \geq \lambda_{0}$. Therefore

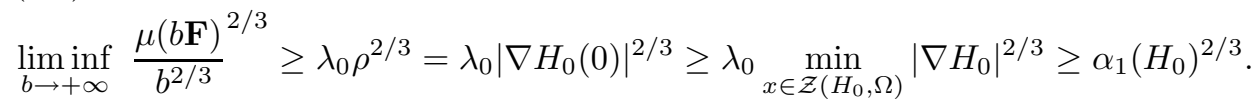

Next assume that $x^{0} \in \partial \Omega$. Then we use a diffeomorphism to straighten a portion of $\partial \Omega$ around $x^{0}$, which gives new local coordinates. Write the equation in these new coordinates. After rescaling and gauge transformations, we find that the limiting 
equation is (1.11), with $\vartheta$ being the angle between $\operatorname{curl}^{2} \mathbf{F}\left(x^{0}\right)$ and the tangent vector $\tau$ of $\partial \Omega$. So we find that

$$
\begin{aligned}
& \liminf _{b \rightarrow+\infty} \frac{\mu(b \mathbf{F})}{b^{2 / 3}} \geq \lambda\left(\mathbb{R}_{+}^{2}, \vartheta\left(x^{0}\right)\right)\left|\nabla H_{0}\left(x^{0}\right)\right|^{2 / 3} \\
& \geq \min _{x \in \mathcal{Z}\left(H_{0}, \partial \Omega\right)} \lambda\left(\mathbb{R}_{+}^{2}, \vartheta(x)\right)\left|\nabla H_{0}(x)\right|^{2 / 3} \geq \alpha_{1}\left(H_{0}\right)^{2 / 3} .
\end{aligned}
$$

Combining (6.2), (6.3) and (6.4), we get the first conclusion in Theorem 2.

Next let $\left\{\hat{x}^{b}\right\} \subset \bar{\Omega}$ be such that

$$
\limsup _{b \rightarrow+\infty} \frac{\left|\psi_{b}\left(\hat{x}^{b}\right)\right|}{\left\|\psi_{b}\right\|_{L^{\infty}(\bar{\Omega})}}>0 .
$$

The above discussion shows that the limiting points of $\left\{\hat{x}^{b}\right\}$ must lie in $\tilde{\mathcal{Z}}\left(H_{0}\right)$. So the second conclusion of Theorem 2 follows.

Proof of Theorem 1. Using Theorem 2 and adopting the arguments in [LP4] (proofs of Theorems 1.1 and 1.3), we obtain Theorem 1. The details are omitted here.

\section{REFERENCES}

[A] S. Agmon, Lectures on exponential decay of solutions of second order elliptic equations: bounds on eigenfunctions of $N$-body Schrödinger operators, Princeton University Press, 1982. MR 85f:35019

[BH] C. Bolley and B. Helffer, An application of semi-classical analysis to the asymptotic study of the supercooling field of a superconducting material, Ann. Inst. Henri Poincaré, Physique Théorique, 58 (1993), 189-233. MR 94k:82120

[BPT] P. Bauman, D. Phillips and Q. Tang, Stable nucleation for the Ginzburg-Landau system with an applied magnetic field, Arch. Rat. Mech. Anal., 142 (1998), 1-43. MR 99g:58040

[BS] A. Bernoff and P. Sternberg, Onset of superconductivity in decreasing fields for general domains, J. Math. Phys. 39 (1998), 1272-1284. MR 99a:82099

[C] S. J. Chapman, Nucleation of superconductivity in OAdecreasing fields, European J. Appl. Math., 5 (1994), part 1, 449-468; part 2, 468-494. MR 95m:82119

[CHO] S. J. Chapman, S. D. Howison and J. R. Ockendon, Macroscopic models for superconductivity, SIAM Review, 34 (1992), 529-560. MR 94b:82037

[DFS] M. del Pino, P. Felmer and P. Sternberg, Boundary concentration for eigenvalue problems related to the onset of superconductivity, Commun. Math. Phys., 210 (2000), 413-446. MR 2001k:35231

[dG] P. G. De Gennes, Superconductivity of Metals and Alloys, W. A. Benjamin, Inc., (1966).

[DGP] Q. Du, M. Gunzburger and J. Peterson, Analysis and approximation of the GinzburgLandau model of superconductivity, SIAM Review, 34 (1992), 45-81. MR 93g:82109

[DH] M. Dauge and B. Helffer, Eigenvalues variation, I, Neumann problem for Sturm-Liouville operators, J. Differential Equations, 104 (1993), 243-262. MR 94j:47097

[GL] V. Ginzburg and L. Landau, On the theory of superconductivity, Zh. Eksper. Teoret. Fiz. 20 (1950), 1064-1082; English transl., L. D. Landau, Collected Papers, Gordon and Breach, New York, 1967, pp. 546-568. MR 38:5577

[GO] M. Gunzburger and J. Ockendon, Mathematical models in superconductivity, SIAM News, November and December (1994).

[GP] T. Giorgi and D. Phillips, The breakdown of superconductivity due to strong fields for the Ginzburg-Landau model, SIAM Journal on Mathematical Analysis, 30 (1999), 341-359. MR 2000b:35235

[H1] B. Helffer, Semi-Classical Analysis for the Schrödinger Operator and Applications, Lecture Notes in Mathematics, vol.1336, Springer-Verlag, 1988. MR 90c:81043

[H2] B. Helffer, Semiclassical analysis for the Schrödinger operator with magnetic wells (after R. Montgomery, B. Helffer-A. Mohamed), pp. 99-114, in: J. Rauch and B. Simon eds., Quasiclassical Methods, The IMA Volumes in Mathematics and Its Applications, vol. 95, Springer, 1997. MR 98m:81034 
[HMoh] B. Helffer and A. Mohamed, Semiclassical analysis for the ground state energy of a Schrödinger operator with magnetic wells, J. Functional Analysis, 138 (1996), 40-81. MR 97h:35177

[HMor] B. Helffer and A. Morame, Magnetic bottles in connection with superconductivity, J. Funct. Anal. 185 (2001), 604-680.

[HP] B. Helffer and Xing-Bin Pan, Upper critical field and location of surface nucleation of superconductivity, Ann. L'Institut Henri Poincaré Analyse Non Linéaire, to appear.

[LP1] Kening Lu and Xing-Bin Pan, The first eigenvalue of Ginzburg-Landau operator, in: Differential Equations and Applications, Bates et al. eds., International Press (1997), 215-226. MR 99j:35205

[LP2] Kening Lu and Xing-Bin Pan, Gauge invariant eigenvalue problems in $\mathbb{R}^{2}$ and in $\mathbb{R}_{+}^{2}$, Trans. Amer. Math. Soc., 352 (2000), 1247-1276. MR 2000j:35248

[LP3] Kening Lu and Xing-Bin Pan, Eigenvalue problems of Ginzburg-Landau operator in bounded domains, J. Math. Phys., 40 (1999), 2647-2670. MR 2001e:35167

[LP4] Kening Lu and Xing-Bin Pan, Estimates of the upper critical field for the GinzburgLandau equations of superconductivity, Physica D, 127 (1-2) (1999), 73-104. MR 2000a:82075

[LP5] Kening Lu and Xing-Bin Pan, Surface nucleation of superconductivity in 3-dimension, J. Differential Equations, 168 (2000), 386-452. MR 2002b:82069

[LP6] Kening Lu and Xing-Bin Pan, Surface nucleation of superconductivity, Methods and Applications of Analysis 8 (2001), 279-300.

[M] R. Montgomery, Hearing the zero locus of a magnetic field, Comm. Math. Phys., 168 (1995), 651-675. MR 96e:81044

[P] Xing-Bin Pan, Upper critical field for superconductivity with edges and corners, Calculus of Variations and PDE's (to appear).

[S] Y. Sibuya, Global theory of a second order linear ordinary differential equation with a polynomial coefficient, North-Holland Mathematics Studies, Vol. 18, North-Holland Publishing Co., Amsterdam-Oxford, 1975. MR 58:6561

[SdG] D. Saint-James and P. G. De Gennes, Onset of superconductivity in decreasing fields, Physics Letters, 6 : (5) (1963), 306-308.

[SST] D. Saint-James and G. Sarma and E.J. Thomas, Type II Superconductivity, Pergamon Press, Oxford, 1969.

[T] M. Tinkham, Introduction to Superconductivity, McGraw-Hill Inc., New York, 1975.

Center for Mathematical Sciences, Zhejiang University, Hangzhou 310027, People's Republic of China; and Department of Mathematics, National University of Singapore, SINGAPORE 119260

E-mail address: matpanxb@nus.edu.sg

Department of Mathematics, National University of Singapore, Singapore 119260

Current address: The Logistics Institute-Asia Pacific National University of Singapore, Singapore 119260 